\title{
Review Article \\ Freeze-Out Parameters in Heavy-Ion Collisions at AGS, SPS, RHIC, and LHC Energies
}

\author{
Sandeep Chatterjee, ${ }^{1,2}$ Sabita Das, ${ }^{3}$ Lokesh Kumar, ${ }^{1,4}$ D. Mishra, ${ }^{1}$ Bedangadas Mohanty, \\ Raghunath Sahoo, ${ }^{5}$ and Natasha Sharma ${ }^{3,6}$ \\ ${ }^{1}$ School of Physical Sciences, National Institute of Science Education and Research, Bhubaneswar 751005, India \\ ${ }^{2}$ Theoretical Physics Division, Variable Energy Cyclotron Centre, Kolkata 700064, India \\ ${ }^{3}$ Institute of Physics, Bhubaneswar 751005, India \\ ${ }^{4}$ Department of Physics, Panjab University, Chandigarh 160014, India \\ ${ }^{5}$ Indian Institute of Technology, Indore 452017, India \\ ${ }^{6}$ University of Tennessee, Knoxville, TN 37996, USA
}

Correspondence should be addressed to Bedangadas Mohanty; bedanga@niser.ac.in

Received 26 July 2014; Revised 17 September 2014; Accepted 18 September 2014

Academic Editor: Bhartendu K. Singh

Copyright (C) 2015 Sandeep Chatterjee et al. This is an open access article distributed under the Creative Commons Attribution License, which permits unrestricted use, distribution, and reproduction in any medium, provided the original work is properly cited. The publication of this article was funded by $\mathrm{SCOAP}^{3}$

\begin{abstract}
We review the chemical and kinetic freeze-out conditions in high energy heavy-ion collisions for AGS, SPS, RHIC, and LHC energies. Chemical freeze-out parameters are obtained using produced particle yields in central collisions while the corresponding kinetic freeze-out parameters are obtained using transverse momentum distributions of produced particles. For chemical freezeout, different freeze-out scenarios are discussed such as single and double/flavor dependent freeze-out surfaces. Kinetic freezeout parameters are obtained by doing hydrodynamic inspired blast wave fit to the transverse momentum distributions. The beam energy and centrality dependence of transverse energy per charged particle multiplicity are studied to address the constant energy per particle freeze-out criteria in heavy-ion collisions.
\end{abstract}

\section{Introduction}

As a result of ultrarelativistic collision between two heavy ions, a fireball is expected to form that rapidly thermalizes. The enormous amount of energy density deposited in the fireball results in large pressure gradients from the central to the peripheral region of the fireball that drives the expansion of the fireball. This expansion leads to cooling of the fireball. Further, as the interparticle distance grows with time, the particles cease to interact after sometime and free stream to the detector. The surface of last scattering is the freeze-out surface. Since scattering could be both elastic (where particle identities do not change) and inelastic (where particle identities change), it is possible to have two distinct freeze-outs, namely, chemical freeze-out (CFO), where inelastic collisions cease, and thermal/kinetic freeze-out (KFO) where elastic collisions cease and the particle mean free path becomes higher than the system size, which forbids the elastic collision of the constituents in the system [1]. While the CFO surface is determined by analysing the measured hadron yields [2-5], the KFO surface can be determined by studying the data of transverse momentum $\left(p_{T}\right)$ distribution of produced particles [6-11]. In general, freezeout could be a complicated process involving duration in time and a hierarchy where different types of particles and reactions switch-off at different times. This leads to the concept of "sequential freeze-out." From kinetic arguments, it is expected that reactions with lower cross-sections switch-off at higher densities/temperature or earlier in time compared to reactions with higher cross-sections. Hence, the chemical freeze-out, which corresponds to inelastic reactions, occurs earlier in time compared to the kinetic freeze-out, which corresponds to elastic reactions. In accordance with the above discussions, one can think of strange or charmed particles 
decoupling from the system earlier than the lighter hadrons. A series of freeze-outs could be thought of corresponding to particular reaction channels [12].

In this review, we present the detailed study of chemical and kinetic freeze-out scenario in central heavy-ion collisions from the lower AGS energies to the largest LHC energies. The paper is organized as follows. In the next section, we discuss chemical freeze-out followed by introduction to chemical freeze-out models employed and then present results and discussions. Section 3 consists of discussions on transverse energy per charged particle and the freeze-out criteria. In Section 4, we discuss the kinetic freeze-out properties obtained using hydrodynamic inspired blast wave model fit to the experimental data. Finally, in Section 5, we give summary and conclusions.

\section{Chemical Freeze-Out}

Quantum Chromodynamics (QCD) which is the quantum field theory that addresses the physics of strong interactions has three conserved charges, namely, baryon number $B$, electric charge $Q$, and strangeness $S$. Thus, the equilibrium thermodynamic state of QCD matter is completely determined by temperature $T_{\mathrm{ch}}$ and the three chemical potentials $\mu_{B}, \mu_{\mathrm{Q}}$, and $\mu_{S}$ corresponding to $B, Q$, and $S$, respectively. Various thermodynamic quantities like pressure, energy density, and so forth, and even different susceptibilities of conserved charges of QCD computed in lattice QCD at zero $\mu_{B}$ compare well with hadron resonance gas (HRG) model predictions for the same up to the quark-hadron transition region around $150-160 \mathrm{MeV}$ [13-15]. Thus, HRG which is a gas of noninteracting hadrons and resonances is a good low $T_{\text {ch }}$ approximation to QCD thermodynamics. These models have also been quite successful in describing the particle multiplicities measured in heavy-ion collision experiments across a wide range of beam energies from $\sqrt{s_{N N}}=2.7-$ $2760 \mathrm{GeV}$ [2-5] with a few thermal parameters to be fitted from data. Apart from heavy ion collisions, even in systems like $e^{+} e^{-}$and $p p$, statistical models have been found to successfully describe the particle multiplicities [16]. For a recent interpretation of such universal behaviour of particle production, please look at [17]. However, recent lattice QCD results on strangeness fluctuations and correlations show that strange hadrons currently unobserved but predicted by quark models and lattice computations can influence the value of the extracted freeze-out temperature within a HRG model approach [18]. For a detailed estimate of the effect of the additional strange hadrons on the freeze-out parameters one needs to model their decay properties. We leave such an exercise for the future. In this review, the hadronic spectrum includes only hadrons listed in the PDG [19].

Out of the three chemical potentials it is a common practice to fix $\mu_{\mathrm{Q}}$ and $\mu_{S}$ from the following constraints:

$$
\begin{aligned}
\frac{\operatorname{Net} B}{\operatorname{Net} Q} & =2.5, \\
\operatorname{Net} S & =0 .
\end{aligned}
$$

Thus, the usual set of free parameters that are fitted to the data are $T_{\mathrm{ch}}, \mu_{B}$, and an overall normalization factor which is the volume $V$. An additional parameter $\gamma_{s}$ called the strangeness suppression factor which accounts for any out of equilibrium production of strangeness is also often used. Such a program allows us to determine the thermodynamic state of the fireball at the time of CFO and hence sets a baseline for all hadronic thermal physics to be pitted against data to isolate signals for quark gluon plasma (QGP) as well as the QCD critical point. Thus, it is necessary to have a very detailed and vivid understanding of the physics of the CFO.

The traditional picture of CFO is where all the hadrons chemically freeze-out together (1CFO). Such a picture provides a descent qualitative description of the hadron multiplicities at all the beam energies with a few exceptions where the $\chi^{2} / \mathrm{NDF}$, where NDF is number of degrees of freedom, is significantly large.

The latest LHC data has posed a serious challenge to this $1 \mathrm{CFO}$ picture where the strange to nonstrange particle ratios like $\Lambda / p$ cannot be explained [20]. This has led to proposals for various alternate freeze-out schemes. In [21], hadronization followed by hadronic afterburner within the hybrid UrQMD model was employed. PYTHIA generated initial condition was followed by hydrodynamic expansion. Then, around energy density of $700 \mathrm{MeV} / \mathrm{fm}^{3}$, using CooperFrye prescription, hadrons were formed and the system entered a transport stage that mainly caused late stage baryon-antibaryon annihilation. This resulted in successful description of all the particle yields. Such an approach has also been employed to successfully describe the centrality dependence of the hadron yields at the LHC [22]. These studies yield a hadronization temperature of $164 \pm 3 \mathrm{MeV}$ for vanishing baryon chemical potential. In another approach, $1 \mathrm{CFO}$ with nonequilibrium quark phase space factors for light and strange quarks were used [23]. This also resulted in good description of the yields.

Both the above approaches accounted for effects on particle yields due to departure from equilibrium physics. In yet another approach, it was realised that one could describe the particle yields within the ambit of equilibrium physics by proposing flavor dependent freeze-out surfaces (2CFO) [24, 25]. It was argued on the basis of hadro-chemistry that strange and nonstrange hadrons could freeze-out at different times (2CFO). In 2CFO, all strange hadrons and those with hidden strangeness freeze-out at the same surface while the rest of the non strange hadrons freeze-out at a separate surface. Here, we will study the dependence of the extracted freeze-out thermal parameters on (a) the choice of the thermodynamic ensemble, (b) choice of the free parameters, (c) choice of particles whose yields are used as inputs to extract the values of the thermal parameters, and (d) choice of the CFO scheme. We find that this program yields fairly robust values of the thermal parameters that show little sensitivity to the specific details of the fitting procedure. Having obtained and studied the systematics of the CFO parameters, we compute several particle ratios and compare with the available data. We find that ratios of particles of unlike flavor are particularly sensitive to the choice of the CFO scheme [26].

2.1. Model. In the most general case, different hadron species would chemically decouple from the fireball at different 
freeze-out surfaces at different times. Thus, the grand partition function $Z$ of all the hadrons that are emitted at different times from the fireball can be written as a product of the partition function at each freeze-out surface as the freezeout at two different surfaces are independent processes. Thus, for a given $\sqrt{s_{N N}}$, the relevant HRG partition function $Z$ in the Grand Canonical Ensemble (GCE) is given by a summed contribution over single particle partition functions $Z_{h}$ of all hadrons:

$$
\begin{gathered}
\log \left[Z\left(\sqrt{s_{N N}}\right)\right]=\sum_{h} \log \left[Z_{h}\right], \\
Z_{h}=Z_{h}\left(T_{h}\left(\sqrt{s_{N N}}\right), \mu_{h}\left(\sqrt{s_{N N}}\right), V_{h}\left(\sqrt{s_{N N}}\right)\right),
\end{gathered}
$$

where $Z_{h}$ is the partition function of the $h$ th hadron. $T_{h}$ and $V_{h}$ are the temperature and volume, respectively, of the fireball as well as the $h$ th hadron at the time of its CFO. In terms of the corresponding fireball chemical potentials $\mu_{B h}, \mu_{\mathrm{Q} h}$, and $\mu_{S h}$, the hadron chemical potential $\mu_{h}$ is expressed as

$$
\mu_{h}=B_{h} \mu_{B h}+Q_{h} \mu_{Q h}+S_{h} \mu_{S h},
$$

where $B_{h}, Q_{h}$, and $S_{h}$ are the quantum numbers of the $h$ th hadron. In 1CFO, there is a single CFO surface and hence $T_{h}\left(\sqrt{s_{N N}}\right)=T\left(\sqrt{s_{N N}}\right)$ for all hadrons. Similarly, $V_{h}, \mu_{B h}$, $\mu_{\mathrm{Q} h}$, and $\mu_{S h}$ are same for all hadrons. In $2 \mathrm{CFO}$, there are two CFO surfaces. $T_{h}=T_{\text {ns }}$ for all nonstrange hadrons while $T_{h}=T_{s}$ for all strange hadrons and those with hidden strangeness content. Volume and chemical potentials are also treated similarly. A partial derivative with respect to $\mu_{h}$ gives the primordial yield of the $h$ th hadron $N_{h}^{p}$ :

$$
\begin{aligned}
N_{h}^{p}= & \frac{\partial}{\partial\left(\mu_{h} / T_{h}\right)} \log [Z] \\
= & \frac{V_{h} T_{h}}{\pi^{2}} g_{h} m_{h}^{2} \sum_{l=1}^{\infty}(-a)^{l+1} l^{-1} K_{2}\left(\frac{l m_{h}}{T_{h}}\right) \\
& \times \exp \left(\frac{l\left(B_{h} \mu_{B h}+Q_{h} \mu_{Q_{h}}+S_{h} \mu_{S h}\right)}{T_{h}}\right),
\end{aligned}
$$

where $a=-1$ for bosons and 1 for fermions and $K_{2}$ is the Bessel function of the second kind. $m_{h}$ and $g_{h}$ are the mass and degeneracy factor of the $h$ th hadron and its conserved charges are $B_{h}, Q_{h}$, and $S_{h}$. The total multiplicity of the $h$ th hadron $N_{h}^{t}$ is given by a sum of the primordial yield as well as feed-down from heavier resonances that decay to it

$$
N_{h}^{t}=N_{h}^{p}+\sum_{j} N_{j}^{p} \times \text { B.R. }_{j \rightarrow h},
$$

where B.R. $j \rightarrow h$ taken from P.D.G. [19] is the branching ratio for the $j$ th hadron to decay to the $h$ th hadron.

The publicly available code THERMUS [27] allows one to compute the hadron yields in 1CFO within the GCE as well as various other ensembles like the canonical ensemble where all the charges are conserved exactly or treat the charges in a mixed ensemble. For example in the Strangeness Canonical
Ensemble (SCE), the baryon and electric charges are conserved on an average in the GCE while strangeness is treated in the canonical ensemble. We have analysed all available hadronic yields and ratios at AGS, SPS, RHIC, and LHC experiments within GCE and SCE with $1 \mathrm{CFO}$ as the freezeout scheme in the THERMUS code. We have also analysed the same data with $2 \mathrm{CFO}$ freeze-out scheme [24]. The unified CFO thermal parameters in $1 \mathrm{CFO}$ lie intermediate to the freeze-out parameters of the strange and nonstrange $\mathrm{CFO}$ surfaces in case of $2 \mathrm{CFO}$. We also report several particle ratios, particularly strange to nonstrange particle ratios that are sensitive to the CFO mechanism chosen.

2.2. Results and Discussions. In Tables 1, 2, 3, and 4, an exhaustive compilation of multiplicity data for the central case available at various beam energies are shown. In addition to the AGS [28-37], SPS [38-46], RHIC data at $\sqrt{s_{N N}}=$ $62.4,130$, and $200 \mathrm{GeV}$ [55-62], and LHC [63] for which the CFO thermal parameters within 1CFO have been already well established, here we have also analysed the preliminary data from RHIC Beam Energy Scan (BES) program [47, 73-78] at $\sqrt{s_{N N}}=7.7,11.5,19.6,27$, and $39 \mathrm{GeV}$ [48-54]. While data for nonstrange and strange mesons and baryons are available for all the energies from SPS onwards, data of the multistrange baryons are not available at the AGS energies (data for $\Lambda$ is only available at the top AGS energies). As we will note later, this has direct consequence on the extraction of the thermal parameters. While $T_{\mathrm{ch}}$ and $\mu_{B}$ seem to be mildly sensitive to the presence of the strange baryons, the extracted value of $\mu_{S}$ seem to lower significantly from the expected trend at the AGS energies due to the absence of the strange baryons from the fits. This is expected since at the AGS energies the fireball is baryon dominated due to large baryon stopping which results in higher values of $\mu_{B}$ as compared to SPS energies [79]. Thus, strange baryons like $\Lambda, \Xi$, and $\Omega$ are expected to play a significant role in determining $\mu_{S}$ as compared to $K$ which is dominant at higher energies where $\mu_{B}$ is small and we have a meson dominated fireball. For all the fits reported here, we always obtain $\mu_{\mathrm{Q}}$ from (1). $\mu_{S}$ and $\gamma_{S}$ are sometimes kept as free parameters while at other times we obtain $\mu_{S}$ from (2) and fix $\gamma_{S}$ to unity. $T_{\mathrm{ch}}, \mu_{B}$, and volume are always treated as free parameters.

Currently, at the BES energies, data for $\pi^{+}, \pi^{-}, K^{+}, K^{-}, p$, $\bar{p}, \Lambda, \bar{\Lambda}, \Xi$, and $\bar{\Xi}$ are available. We use the same particles for the SPS, top RHIC, and LHC energies for a uniform treatment at all energies. The extracted thermal parameters (a) $T_{\mathrm{ch}}$, (b) $\mu\left(\mu_{B}, \mu_{S}\right.$, and $\left.\mu_{\mathrm{Q}}\right),(\mathrm{c}) \gamma_{s}$, and (d) radius, which is reflective of volume of the system, are shown in Figure 1. Results for both SCE and GCE are shown for comparison. We find that the results are quite similar in the two ensembles studied. However, as seen in Figure 5, the $\chi^{2} / \mathrm{NDF}$ is consistently lower for SCE than GCE at all energies mainly because there is one less parameter to fit in case of SCE. We have also repeated the same exercise using ratios instead of yields. We found that though the extracted $T_{\mathrm{ch}}$ and $\mu_{B}$ parameters are consistent within errors for the two cases, in general the central values for $T_{\mathrm{ch}}\left(\mu_{B}\right)$ are higher (lower) when particle yields are used for fitting compared to the case when particle ratios are used. The $\chi^{2} / \mathrm{NDF}$ is reduced in ratio case as the uncertainty over 
TABLE 1: Details of the AGS data sets used for fit with references.

\begin{tabular}{|c|c|c|c|c|c|c|}
\hline$\sqrt{s_{N N}}(\mathrm{GeV})$ & Expt. & System & Cent. (\%) & Particles $(d N / d y)$ & Antiparticles $(d N / d y)$ & Reference \\
\hline \multirow{4}{*}{2.7} & E866, E895 & \multirow{4}{*}{$\mathrm{Au}-\mathrm{Au}$} & \multirow{4}{*}{$0-5$} & $\pi^{+}: 14.1 \pm 2.17$ & $\pi^{-}: 21.3 \pm 1.3$ & {$[28,29]$} \\
\hline & E866 & & & $K^{+}: 0.381 \pm 0.059$ & & {$[30]$} \\
\hline & E895 & & & $p: 82.2 \pm 8.2$ & & {$[31]$} \\
\hline & E895 & & & $\Lambda: 0.76 \pm 0.082$ & & {$[32]$} \\
\hline \multirow{4}{*}{3.32} & E866, E895 & \multirow{4}{*}{$\mathrm{Au}-\mathrm{Au}$} & \multirow{4}{*}{$0-5$} & $\pi^{+}: 26.4 \pm 3.97$ & $\pi^{-}: 39 \pm 2.1$ & {$[28,29]$} \\
\hline & E866 & & & $K^{+}: 2.34 \pm 0.35$ & $K^{-}: 0.19 \pm 0.03$ & [30] \\
\hline & E895 & & & $p: 72.6 \pm 7.1$ & & {$[31]$} \\
\hline & E895 & & & $\Lambda: 3.6 \pm 0.37$ & & {$[32]$} \\
\hline \multirow{4}{*}{3.84} & E866, E895 & \multirow{4}{*}{$\mathrm{Au}-\mathrm{Au}$} & \multirow{4}{*}{$0-5$} & $\pi^{+}: 38.9 \pm 5.85$ & $\pi^{-}: 50.8 \pm 2.7$ & {$[28,29]$} \\
\hline & E895 & & & $K^{+}: 4.84 \pm 0.74$ & $K^{-}: 0.61 \pm 0.09$ & {$[30]$} \\
\hline & E895 & & & $p: 63 \pm 6.11$ & & {$[31]$} \\
\hline & E895 & & & $\Lambda: 6.8 \pm 0.71$ & & {$[32]$} \\
\hline \multirow{4}{*}{4.3} & E866, E895 & \multirow{4}{*}{$\mathrm{Au}-\mathrm{Au}$} & \multirow{4}{*}{$0-5$} & $\pi^{+}: 49.7 \pm 7.5$ & $\pi^{-}: 61.1 \pm 3.4$ & {$[28,29]$} \\
\hline & E895 & & & $K^{+}: 7.85 \pm 1.2$ & $K^{-}: 1.26 \pm 0.2$ & {$[30]$} \\
\hline & E895 & & & $p: 64.1 \pm 6.3$ & & {$[33]$} \\
\hline & E895 & & & $\Lambda: 10.25 \pm 1.1$ & & {$[32]$} \\
\hline \multirow{5}{*}{4.85} & E866, E895 & \multirow{5}{*}{$\mathrm{Au}-\mathrm{Au}$} & \multirow{5}{*}{$0-5$} & $\pi^{+}: 57.1 \pm 8.6$ & $\pi^{-}: 67 \pm 6.77$ & {$[28,34]$} \\
\hline & E895 & & & $K^{+}: 11.55 \pm 1.74$ & $K^{-}: 2.21 \pm 0.33$ & [30] \\
\hline & E895 & & & $p: 61 \pm 6.2$ & $\bar{p}: 0.02 \pm 0.002$ & {$[35,36]$} \\
\hline & E891 & & & $\Lambda: 10 \pm 1$ & $\bar{\Lambda}: 0.02 \pm 0.005$ & {$[32,36]$} \\
\hline & E891 & & & $\phi: 0.362 \pm 0.085$ & & [37] \\
\hline
\end{tabular}

volume is irrelevant. However, it is not advisable to use ratios for fitting as the specific choice of independent ratios out of various possibilities could bias the results of the fits [80]. This will become more clear from our later discussion on particle ratios.

In the above case we had treated $\mu_{S}$ as a free parameter and extracted it from fits to data. One could also impose strangeness neutrality condition as in (2) and extract $\mu_{S}$ from it. We had also allowed $\gamma_{s}$ to be a free parameter. However, as seen from Figure 1, $\gamma_{s}$ does not seem to show any systematic dependence with $\sqrt{s_{N N}}$ and hovers around unity. We thus repeated the fits for two further different parameter sets. In one case we still allowed $\gamma_{s}$ to be free while fixed $\mu_{S}$ from (2) while in the other case we fixed $\gamma_{s}=1$ in addition to fixing $\mu_{S}$. We have compared these three cases in Figure 2 . While the thermal parameters extracted seem to be very robust and hence insensitive to the different fitting procedures, $\chi^{2} / \mathrm{NDF}$ is least for the case where $\gamma_{s}$ is held at unity and $\mu_{S}$ is solved from the constraint (2). This is mainly because this case has the least number of free parameters and hence NDF is the largest.

So far we have been working with only $\pi^{+}, \pi^{-}, K^{+}$, $K^{-}, p, \bar{p}, \Lambda, \bar{\Lambda}, \Xi$, and $\bar{\Xi}$ for uniform treatment. Having studied the dependence on the choice of ensembles and fitting procedures with the above uniform particle set at all available energies, we now extend our input particle yield database to all available particles like $\phi$ and $\Omega$ at all energies. In Figure 3 , we have presented the above comparison within the GCE with $\gamma_{s}=1$ and $\mu_{S}$ and $\mu_{Q}$ fixed from the constraints given by (1) and (2). Overall, the thermal parameters show mild dependence on the inclusion of $\phi$ and $\Omega$. At AGS energies, the extracted $\mu_{Q}$ and $\mu_{S}$ fall short of the expected value from the SPS trend due to the absence of the strange baryons from the input database.

Thus, in general, the extracted thermal parameters are quite robust and insensitive to details like choice of ensembles, fitting procedures and whether or not particles like $\phi$ and $\Omega$ are included in the fits. All the above fits were done within 1CFO. As seen from Figure 5, $\chi^{2} / \mathrm{NDF}$ which is a measure of the goodness of fits is quite large at few energies. This has led to efforts to develop new freeze-out schemes which can describe the yields better. One such development which we will discuss here is the $2 \mathrm{CFO}$ scheme of CFO. We have already discussed the $2 \mathrm{CFO}$ scheme in detail in Section 2.1. In Figure 4 , we have presented the thermal parameters extracted in the $2 \mathrm{CFO}$ scheme and compared with those of 1CFO in the SCE with $\gamma_{s}=1$ and $\mu_{Q}$ solved from (1). At all the energies we find that the nonstrange freeze-out temperature $T_{n s}$ is consistently lower than the strange freeze-out temperature $T_{s}$. While at the LHC, $T_{s}$, and $T_{n s}$ differ by about $5 \%$, at the top SPS energy they differ by about $15 \%$. This can be interpreted as an early freeze-out for the strange hadrons [24, 83]. The 1CFO thermal parameters lie intermediate to the corresponding $2 \mathrm{CFO}$ values for the nonstrange and strange CFO surfaces. There is substantial improvement in $\chi^{2} / \mathrm{NDF}$ for $2 \mathrm{CFO}$ as compared to $1 \mathrm{CFO}$.

Now we will look at how well we can describe the different particle ratios within $1 \mathrm{CFO}$ and $2 \mathrm{CFO}$ freeze-out schemes. 
TABLE 2: Details of the SPS data sets used for fit with references.

\begin{tabular}{|c|c|c|c|c|c|c|}
\hline$\sqrt{s_{N N}}(\mathrm{GeV})$ & Expt. & System & Cent. (\%) & Particles $(d N / d y)$ & Antiparticles $(d N / d y)$ & Reference \\
\hline \multirow{6}{*}{6.27} & \multirow[t]{6}{*}{ NA49 } & \multirow{6}{*}{$\mathrm{Pb}-\mathrm{Pb}$} & \multirow{6}{*}{$0-7$} & $\pi^{+}: 72.9 \pm 3.6$ & $\pi^{-}: 84.8 \pm 4.2$ & {$[38]$} \\
\hline & & & & $K^{+}: 16.4 \pm 0.7$ & $K^{-}: 5.58 \pm 0.13$ & {$[38]$} \\
\hline & & & & $p: 46.1 \pm 5.07$ & $\bar{p}: 0.06 \pm 0.012$ & {$[39]$} \\
\hline & & & & $\Lambda: 13.4 \pm 1.1$ & $\bar{\Lambda}: 0.1 \pm 0.02$ & {$[40]$} \\
\hline & & & & $\Xi^{-}: 0.93 \pm 0.16$ & & {$[40]$} \\
\hline & & & & $\phi: 1.17 \pm 0.44$ & & {$[41]$} \\
\hline \multirow{6}{*}{7.62} & \multirow[t]{6}{*}{ NA49 } & \multirow{6}{*}{$\mathrm{Pb}-\mathrm{Pb}$} & \multirow{6}{*}{$0-7$} & $\pi^{+}: 83 \pm 4.2$ & $\pi^{-}: 96.5 \pm 4.83$ & {$[38]$} \\
\hline & & & & $K^{+}: 21.2 \pm 1.7$ & $K^{-}: 7.8 \pm 0.22$ & {$[38]$} \\
\hline & & & & $p: 42.1 \pm 4.7$ & $\bar{p}: 0.16 \pm 0.03$ & [39] \\
\hline & & & & $\Lambda: 14.7 \pm 1.2$ & $\bar{\Lambda}: 0.21 \pm 0.03$ & {$[40]$} \\
\hline & & & & $\Xi^{-}: 1.17 \pm 0.18$ & $\bar{\Xi}^{+}: 0.05 \pm 0.014$ & {$[40]$} \\
\hline & & & & $\phi: 0.94 \pm 0.33$ & & {$[41]$} \\
\hline \multirow{7}{*}{8.76} & \multirow[t]{7}{*}{ NA49 } & \multirow{7}{*}{$\mathrm{Pb}-\mathrm{Pb}$} & \multirow{7}{*}{$0-7$} & $\pi^{+}: 96.6 \pm 6.01$ & $\pi^{-}: 106.1 \pm 6.01$ & {$[42]$} \\
\hline & & & & $K^{+}: 20.1 \pm 1.04$ & $K^{-}: 7.58 \pm 0.42$ & {$[42]$} \\
\hline & & & & $p: 41.3 \pm 4.28$ & $\bar{p}: 0.32 \pm 0.04$ & {$[39]$} \\
\hline & & & & $\Lambda: 14.6 \pm 1.22$ & $\bar{\Lambda}: 0.33 \pm 0.04$ & {$[40]$} \\
\hline & & & & $\Xi^{-}: 1.15 \pm 0.17$ & $\bar{\Xi}^{+}: 0.07 \pm 0.01$ & {$[40]$} \\
\hline & & & & $\phi: 1.16 \pm 0.21$ & & {$[41]$} \\
\hline & & & & $\Omega^{-}: 0.1 \pm 0.03$ & & {$[43]$} \\
\hline \multirow{6}{*}{12.3} & \multirow[t]{6}{*}{ NA49 } & \multirow{6}{*}{$\mathrm{Pb}-\mathrm{Pb}$} & \multirow{6}{*}{$0-7$} & $\pi^{+}: 132 \pm 7.02$ & $\pi^{-}: 140.4 \pm 7.02$ & {$[42]$} \\
\hline & & & & $K^{+}: 24.6 \pm 1.2$ & $K^{-}: 11.7 \pm 0.6$ & {$[42]$} \\
\hline & & & & $p: 30.1 \pm 3.2$ & $\bar{p}: 0.87 \pm 0.11$ & {$[44]$} \\
\hline & & & & $\Lambda: 12.9 \pm 1.02$ & $\bar{\Lambda}: 0.82 \pm 0.09$ & {$[45]$} \\
\hline & & & & $\Xi^{-}: 1.22 \pm 0.19$ & $\bar{\Xi}^{+}: 0.21 \pm 0.04$ & {$[43]$} \\
\hline & & & & $\phi: 1.52 \pm 0.25$ & & {$[46]$} \\
\hline \multirow{7}{*}{17.3} & \multirow[t]{6}{*}{ NA49 } & \multirow{6}{*}{$\mathrm{Pb}-\mathrm{Pb}$} & \multirow{6}{*}{$0-7$} & $\pi^{+}: 170 \pm 9.03$ & $\pi^{-}: 175.4 \pm 9.03$ & {$[42]$} \\
\hline & & & & $K^{+}: 29.6 \pm 1.53$ & $K^{-}: 16.8 \pm 0.82$ & {$[42]$} \\
\hline & & & & $p: 29.6 \pm 3.1$ & $\bar{p}: 1.66 \pm 0.24$ & {$[44]$} \\
\hline & & & & $\Lambda: 10.3 \pm 1.1$ & $\bar{\Lambda}: 1.34 \pm 0.14$ & {$[45]$} \\
\hline & & & & $\Xi^{-}: 1.56 \pm 0.19$ & $\bar{\Xi}^{+}: 0.35 \pm 0.04$ & {$[43]$} \\
\hline & & & & $\phi: 2.44 \pm 0.13$ & & {$[46]$} \\
\hline & NA57 & & & $\Omega^{-}: 0.19 \pm 0.03$ & $\bar{\Omega}^{+}: 0.097 \pm 0.02$ & {$[43]$} \\
\hline
\end{tabular}

As argued in [26], strange to nonstrange particle ratios are particularly sensitive to the choice of CFO scheme. This is easily understood from the following expression for the particle ratios in HRG

$$
\begin{aligned}
\frac{N_{i}}{N_{j}}= & \frac{g_{i} V_{i}}{g_{j} V_{j}}\left(\frac{T_{i} m_{i}}{T_{j} m_{j}}\right)^{3 / 2} \exp \left(\frac{m_{j}}{T_{j}}-\frac{m_{i}}{T_{i}}\right) \\
& \times \exp \left(\frac{B_{i} \mu_{B i}}{T_{i}}-\frac{B_{j} \mu_{B j}}{T_{j}}\right) \\
& \times \exp \left(\frac{Q_{i} \mu_{Q_{i}}}{T_{i}}-\frac{Q_{j} \mu_{Q_{j}}}{T_{j}}\right) \\
& \times \exp \left(\frac{S_{i} \mu_{S_{i}}}{T_{i}}-\frac{S_{j} \mu_{S j}}{T_{j}}\right) .
\end{aligned}
$$

The above equation is obtained from (5) by taking the asymptotic limit $m / T \gg 1$. It is clearly evident from (7) that for ratios of particles of unlike flavors, the thermal factor coefficient $\left(V_{i} T_{i}^{3 / 2}\right) /\left(V_{j} T_{j}^{3 / 2}\right)$ does not cancel off while in $1 \mathrm{CFO}$ this additional factor does not arise at all. Thus, ratios of particles of unlike flavor are good probes to distinguish different freeze-out schemes. In Figure 6, we have compared the particle ratios as obtained in 1CFO and 2CFO models of $\mathrm{CFO}$ and compared them with data. The data for ratios are obtained from the ratios of the data of corresponding yields while the errors are computed in quadratures. As argued above, we see that particle ratios of unlike flavor like $K / \pi$ and $\Lambda / p$ discriminate between different CFO schemes while those of same flavor like $p / \pi$ and $\Lambda / K$ appear similar in both the schemes. We also find $2 \mathrm{CFO}$ to describe all the ratios better as compared to $1 \mathrm{CFO}$.

We have also plotted a triple ratio $(\Lambda / \bar{\Lambda}) /\left((p / \bar{p})\left(K^{-} / K^{+}\right)\right)$ in Figure 6. Since the total $B, Q$ and $S$ charges carried by $\Lambda$ are same as those carried by $p$ and $K$ together, the fugacity factors cancel off and this ratio is to a very good approximation unity in 1CFO [84]. However, in 2CFO there is no such 
TABLE 3: Details of the RHIC data sets used for fit with references.

\begin{tabular}{|c|c|c|c|c|c|c|}
\hline$\sqrt{s_{N N}}(\mathrm{GeV})$ & Expt. & System & Cent. (\%) & Particles $(d N / d y)$ & Antiparticles $(d N / d y)$ & Reference \\
\hline \multirow{5}{*}{7.7} & \multirow{5}{*}{ STAR } & \multirow{5}{*}{$\mathrm{Au}-\mathrm{Au}$} & $0-5$ & $\pi^{+}: 93.4 \pm 8.4$ & $\pi^{-}: 100 \pm 9.03$ & {$[47-50]$} \\
\hline & & & & $K^{+}: 20.8 \pm 1.7$ & $K^{-}: 7.7 \pm 0.62$ & {$[47-50]$} \\
\hline & & & & $p: 54.96 \pm 6.6$ & $\bar{p}: 0.39 \pm 0.06$ & {$[47-50]$} \\
\hline & & & & $\Lambda: 16.7 \pm 1.71$ & $\bar{\Lambda}: 0.18 \pm 0.02$ & {$[47,49-52]$} \\
\hline & & & & $\Xi^{-}: 1.17 \pm 0.12$ & $\bar{\Xi}^{+}: 0.08 \pm 0.01$ & {$[47,49-52]$} \\
\hline \multirow{5}{*}{11.5} & \multirow{5}{*}{ STAR } & \multirow{5}{*}{$\mathrm{Au}-\mathrm{Au}$} & $0-5$ & $\pi^{+}: 123.9 \pm 12.4$ & $\pi^{-}: 129.8 \pm 13.01$ & {$[47-50]$} \\
\hline & & & & $K^{+}: 25 \pm 2.5$ & $K^{-}: 12.3 \pm 1.24$ & {$[47-50]$} \\
\hline & & & & $p: 44 \pm 5.7$ & $\bar{p}: 1.46 \pm 0.2$ & [47-50] \\
\hline & & & & $\Lambda: 14.97 \pm 1.52$ & $\bar{\Lambda}: 0.72 \pm 0.07$ & {$[47,49-52]$} \\
\hline & & & & $\Xi^{-}: 1.59 \pm 0.17$ & $\bar{\Xi}^{+}: 0.19 \pm 0.02$ & {$[47,49-52]$} \\
\hline \multirow{5}{*}{19.6} & \multirow{5}{*}{ STAR } & \multirow{5}{*}{$\mathrm{Au}-\mathrm{Au}$} & $0-5$ & $\pi^{+}: 161.4 \pm 17.8$ & $\pi^{-}: 165.8 \pm 18.3$ & [53] \\
\hline & & & & $K^{+}: 29.56 \pm 2.97$ & $K^{-}: 18.8 \pm 1.89$ & {$[53]$} \\
\hline & & & & $p: 34.2 \pm 4.5$ & $\bar{p}: 4.16 \pm 0.58$ & {$[53]$} \\
\hline & & & & $\Lambda: 13.5 \pm 1.36$ & $\bar{\Lambda}: 1.98 \pm 0.19$ & {$[52]$} \\
\hline & & & & $\Xi^{-}: 1.77 \pm 0.18$ & $\bar{\Xi}^{+}: 0.49 \pm 0.05$ & {$[52]$} \\
\hline \multirow{5}{*}{27} & \multirow{5}{*}{ STAR } & \multirow{5}{*}{$\mathrm{Au}-\mathrm{Au}$} & $0-5$ & $\pi^{+}: 172.9 \pm 19.02$ & $\pi^{-}: 177.1 \pm 19.5$ & {$[54]$} \\
\hline & & & & $K^{+}: 31.1 \pm 1.9$ & $K^{-}: 22.6 \pm 2.3$ & {$[54]$} \\
\hline & & & & $p: 31.7 \pm 4.1$ & $\bar{p}: 6 . \pm 0.78$ & {$[54]$} \\
\hline & & & & $\Lambda: 12.5 \pm 1.26$ & $\bar{\Lambda}: 2.91 \pm 0.29$ & {$[52]$} \\
\hline & & & & $\Xi^{-}: 1.81 \pm 0.18$ & $\bar{\Xi}^{+}: 0.68 \pm 0.07$ & {$[52]$} \\
\hline \multirow{5}{*}{39} & \multirow{5}{*}{ STAR } & \multirow{5}{*}{$\mathrm{Au}-\mathrm{Au}$} & $0-5$ & $\pi^{+}: 182.3 \pm 20.1$ & $\pi^{-}: 185.8 \pm 20.5$ & {$[47-50]$} \\
\hline & & & & $K^{+}: 31.9 \pm 2.9$ & $K^{-}: 25 . \pm 2.3$ & {$[47-50]$} \\
\hline & & & & $p: 26.57 \pm 2.92$ & $\bar{p}: 8.49 \pm 1.02$ & {$[47-50]$} \\
\hline & & & & $\Lambda: 11.2 \pm 1.13$ & $\bar{\Lambda}: 3.92 \pm 0.39$ & {$[47,49-52]$} \\
\hline & & & & $\Xi^{-}: 1.62 \pm 0.17$ & $\bar{\Xi}^{+}: 0.83 \pm 0.08$ & {$[47,49-52]$} \\
\hline \multirow{7}{*}{62.4} & \multirow{7}{*}{ STAR } & \multirow{7}{*}{$\mathrm{Au}-\mathrm{Au}$} & $0-5$ & $\pi^{+}: 233 \pm 17$ & $\pi^{-}: 237 \pm 17$ & [55] \\
\hline & & & & $K^{+}: 37.6 \pm 2.7$ & $K^{-}: 32.4 \pm 2.3$ & {$[55]$} \\
\hline & & & & $p: 29 \pm 3.8$ & $\bar{p}: 13.6 \pm 1.7$ & {$[55]$} \\
\hline & & & & $\Lambda: 15.7 \pm 2.3$ & $\bar{\Lambda}: 8.3 \pm 1.1$ & {$[56]$} \\
\hline & & & & $\Xi^{-}: 1.63 \pm 0.18$ & $\bar{\Xi}^{+}: 1.03 \pm 0.11$ & {$[56]$} \\
\hline & & & $0-20$ & $\phi: 3.52 \pm 0.45$ & & {$[57]$} \\
\hline & & & $0-20$ & $\Omega^{-}: 0.212 \pm 0.033$ & $\bar{\Omega}^{+}: 0.167 \pm 0.031$ & {$[56]$} \\
\hline \multirow{7}{*}{130} & \multirow{7}{*}{ STAR } & \multirow{7}{*}{$\mathrm{Au}-\mathrm{Au}$} & $0-5$ & $\pi^{+}: 278 \pm 20$ & $\pi^{-}: 280 \pm 20$ & [55] \\
\hline & & & & $K^{+}: 46.3 \pm 3$ & $K^{-}: 42.7 \pm 2.8$ & {$[55]$} \\
\hline & & & & $p: 28.2 \pm 3.1$ & $\bar{p}: 20 \pm 2.2$ & {$[55]$} \\
\hline & & & & $\Lambda: 17 \pm 1.75$ & $\bar{\Lambda}: 12.3 \pm 1.24$ & {$[58]$} \\
\hline & & & $0-10$ & $\Xi^{-}: 2 \pm 0.24$ & $\bar{\Xi}^{+}: 1.7 \pm 0.21$ & [59] \\
\hline & & & $0-10$ & $\phi: 5.73 \pm 0.783$ & & {$[60]$} \\
\hline & & & $0-20$ & $\Omega^{-}: 0.32 \pm 0.1$ & $\bar{\Omega}^{+}: 0.34 \pm 0.1$ & {$[56]$} \\
\hline \multirow{7}{*}{200} & & & $0-5$ & $\pi^{+}: 322 \pm 25$ & $\pi^{-}: 327 \pm 25$ & [55] \\
\hline & & & & $K^{+}: 51.3 \pm 6.5$ & $K^{-}: 49.5 \pm 6.2$ & {$[55]$} \\
\hline & & & & $p: 34.7 \pm 4.4$ & $\bar{p}: 26.7 \pm 3.4$ & {$[55]$} \\
\hline & STAR & $\mathrm{Au}-\mathrm{Au}$ & & $\Lambda: 16.7 \pm 1.1$ & $\bar{\Lambda}: 12.7 \pm 0.9$ & {$[61]$} \\
\hline & & & & $\Xi^{-}: 2.17 \pm 0.2$ & $\bar{\Xi}^{+}: 1.83 \pm 0.2$ & {$[61]$} \\
\hline & & & $0-10$ & $\phi: 7.42 \pm 0.69$ & & {$[62]$} \\
\hline & & & $0-20$ & $\Omega^{-}: 0.31 \pm 0.06$ & $\bar{\Omega}^{+}: 0.33 \pm 0.07$ & {$[56]$} \\
\hline
\end{tabular}


TABLE 4: Details of the data on hadron yields available at the different $\sqrt{s_{N N}}$ for the central case only.

\begin{tabular}{|c|c|c|c|c|c|c|}
\hline$\sqrt{s_{N N}}(\mathrm{GeV})$ & Expt. & System & Cent. (\%) & Particles $(d N / d y)$ & Antiparticles $(d N / d y)$ & Reference \\
\hline \multirow{7}{*}{2760} & \multirow{7}{*}{ ALICE } & \multirow{7}{*}{$\mathrm{Pb}-\mathrm{Pb}$} & \multirow{7}{*}{$0-10$} & $\pi^{+}: 669.5 \pm 48$ & $\pi^{-}: 668 \pm 47$ & {$[63]$} \\
\hline & & & & $K^{+}: 100 \pm 8$ & $K^{-}: 99.5 \pm 8.51$ & [63] \\
\hline & & & & $p: 31 \pm 2.5$ & $\bar{p}: 30.5 \pm 2.5$ & [63] \\
\hline & & & & $\Lambda: 24.1 \pm 2.6$ & & [63] \\
\hline & & & & $\Xi^{-}: 3.34 \pm 0.25$ & $\bar{\Xi}^{+}: 3.28 \pm 0.28$ & [63] \\
\hline & & & & $\phi: 12.8 \pm 1.5$ & & [63] \\
\hline & & & & $\Omega^{-}: 0.58 \pm 0.10$ & $\bar{\Omega}^{+}: 0.60 \pm 0.11$ & [63] \\
\hline
\end{tabular}
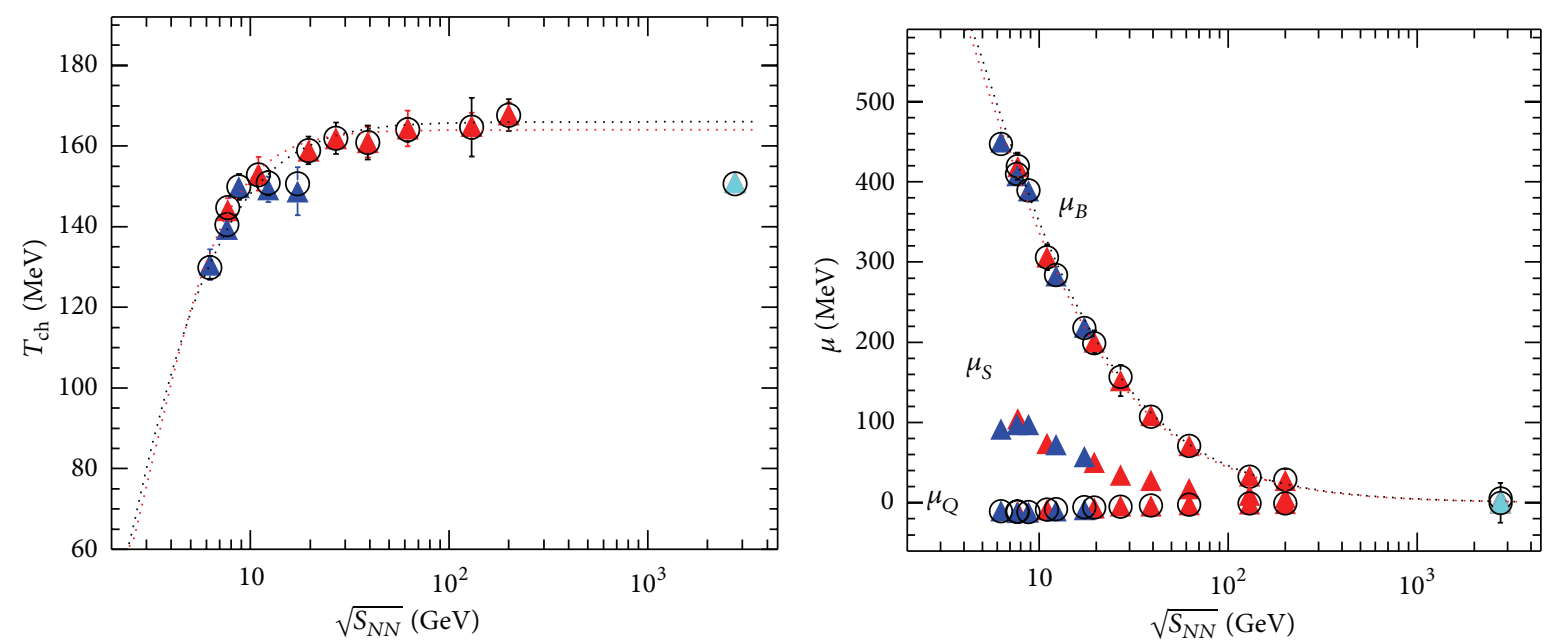

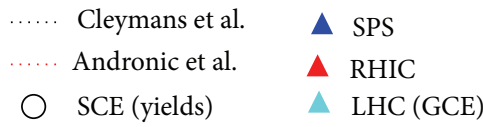

(a)

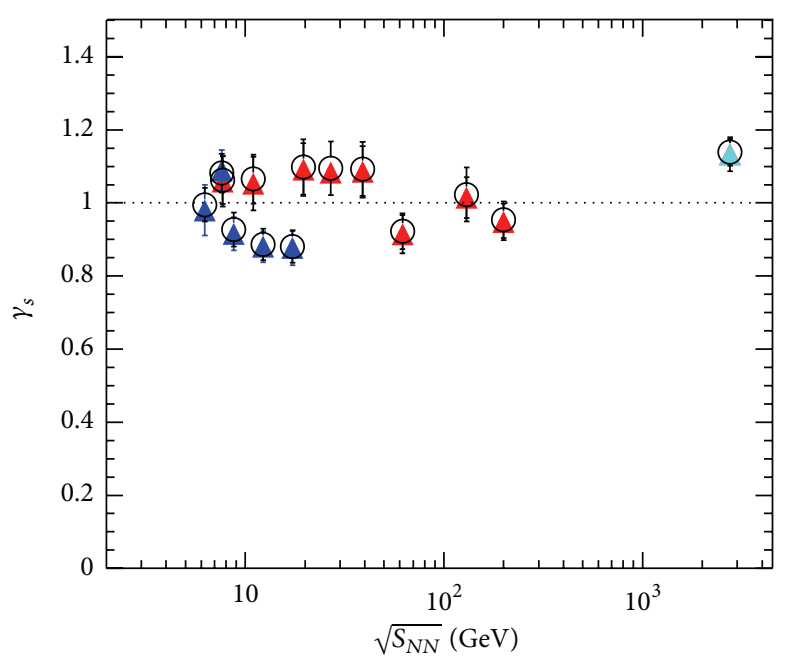

SCE (yields)

$\triangle$ SPS
- RHIC

AHC (GCE)
SCE (yields) Cleymans et al. Andronic et al.

$\Delta$ SPS

- RHIC

$\triangle$ LHC (GCE)

(b)

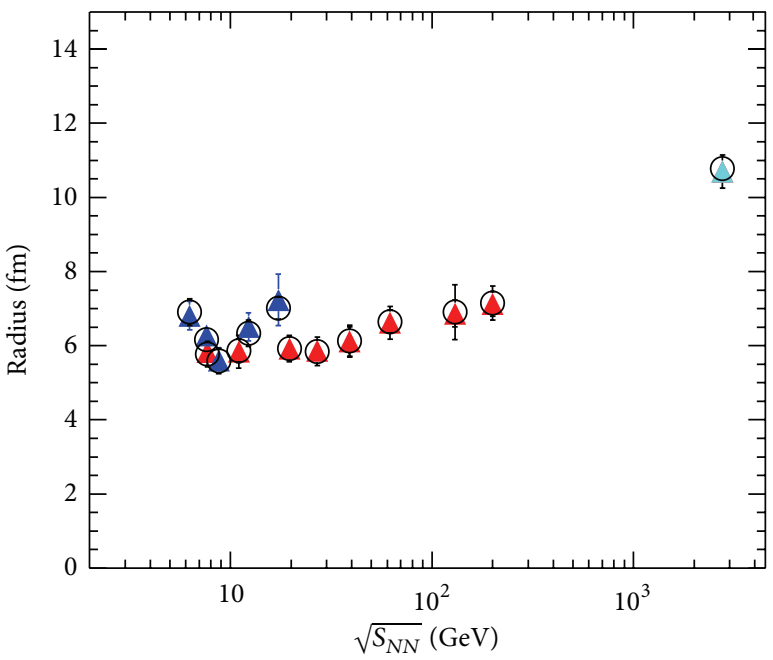

$\begin{array}{ll}\bigcirc \text { SCE (yields) } & \Delta \text { RHIC } \\ \Delta \text { SPS } & \Delta \text { LHC (GCE) }\end{array}$

(d)

Figure 1: Dependence on the Choice of Ensemble. Thermal parameters (a) $T_{\mathrm{ch}}$, (b) $\mu\left(\mu_{B}, \mu_{S}\right.$, and $\left.\mu_{\mathrm{Q}}\right)$, (c) $\gamma_{s}$, and (d) radius, plotted versus collision energy, extracted within the GCE and SCE ensembles with 1CFO as the freeze-out scheme. The dotted curves in the figures represent parameterizations from $[81,82]$. The choice of ensemble does not seem to influence the values of the extracted thermal parameters. However, the $\chi^{2} / \mathrm{NDF}$, which is similar for SPS and higher energies, is significantly smaller for SCE at the AGS energies. This is mainly because in the SCE there is one less free parameter. 

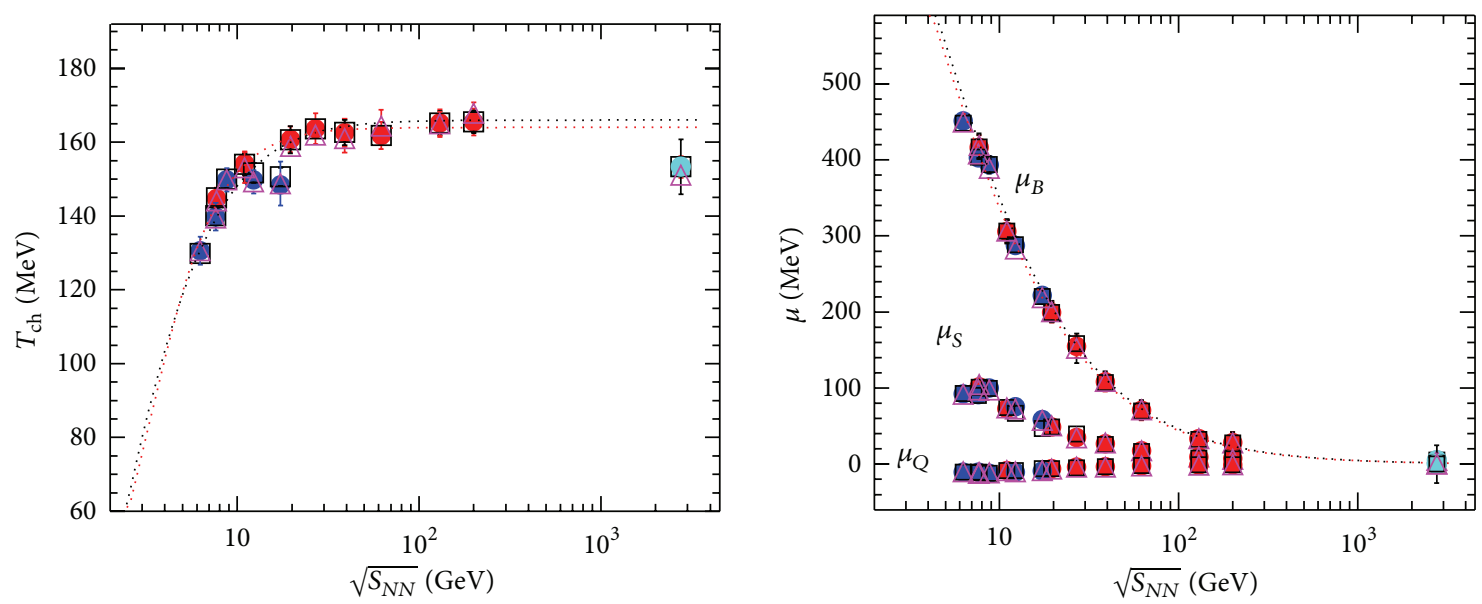

$$
\begin{array}{ll}
\triangle \mu_{\mathrm{Q}}=B / 2 \mathrm{Q} & \text { RHIC } \\
-\mu_{\mathrm{Q}}=B / 2 \mathrm{Q} \text { and } \gamma_{s}=1 & \text { SPS } \\
\square \mu_{\mathrm{Q}}=B / 2 \mathrm{Q}, \mu_{\mathrm{S}}=0 \text { and } \gamma_{s}=1 & \text { LHC (yields, GCE) } \\
\ldots \ldots \text { Cleymans et al. } & \\
\ldots . . . \text { Andronic et al. } &
\end{array}
$$
$\triangle \mu_{\mathrm{Q}}=B / 2 \mathrm{Q}$
$\mu_{\mathrm{Q}}=B / 2 \mathrm{Q}$ and $\gamma_{s}=1$
- RHIC
$\square \mu_{\mathrm{Q}}=B / 2 \mathrm{Q}, \mu_{\mathrm{S}}=0$ and $\gamma_{s}=1 \odot$ LHC (yields, GCE)
..... Cleymans et al.
Andronic et al.

(b)

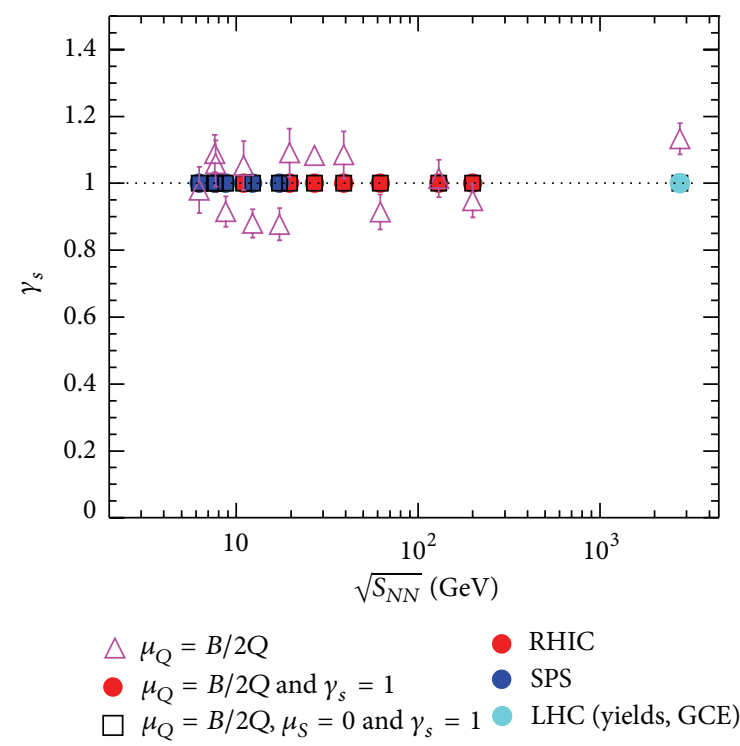

(c)

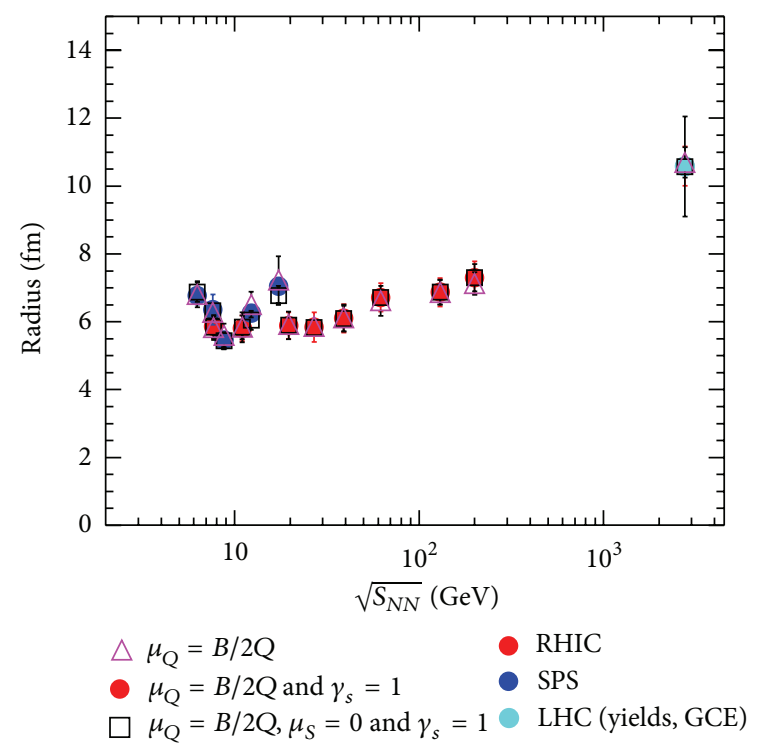

(d)

Figure 2: Dependence on the Fitting Procedure. Comparative study of the thermal parameters (a) $T_{\mathrm{ch}}$, (b) $\mu$ ( $\mu_{B}, \mu_{S}$, and $\mu_{\mathrm{Q}}$ ), (c) $\gamma_{s}$, and (d) radius, plotted versus collision energy, extracted within GCE with $1 \mathrm{CFO}$ as the freeze-out scheme in the three cases: both $\mu_{S}$ and $\gamma_{s}$ free, $\gamma_{s}=1$ and $\mu_{S}$ free, and in the third case $\gamma_{s}=1$ and $\mu_{S}$ fixed from (2). The fitted parameters are insensitive to the chosen scheme.

constraint. We find in data appreciable deviation from unity below $\sqrt{s_{N N}}=10 \mathrm{GeV}$ which can not be described in 1CFO. Currently the errors are very large and hence it is difficult to conclude anything definite. The main reason behind this is that the errors have been computed in quadratures. This might have led to an overestimate of the errors. A proper estimate of the errors of these quantities by taking into account correlations among various sources of errors is highly desired from experimental side.
With the availability of experimental data on light nuclei yields, we can also test the CFO (2CFO and 1CFO/ THERMUS) model calculations for the light nuclei production. Figure 7 shows the ratio of light nuclei as a function of energy $\sqrt{s_{N N}}$. Figure $7(\mathrm{a})$ is for $d / p$ ratio and Figure $7(\mathrm{~b})$ is for $\frac{3}{\Lambda} \bar{H} / 3 \overline{H e}$. The solid circles denote the experimental data and bands are used to represent model calculations. In case of THERMUS calculation, the chemical freeze-out temperature $\left(T_{\text {ch }}\right)$ and baryon chemical potential $\left(\mu_{B}\right)$ are 


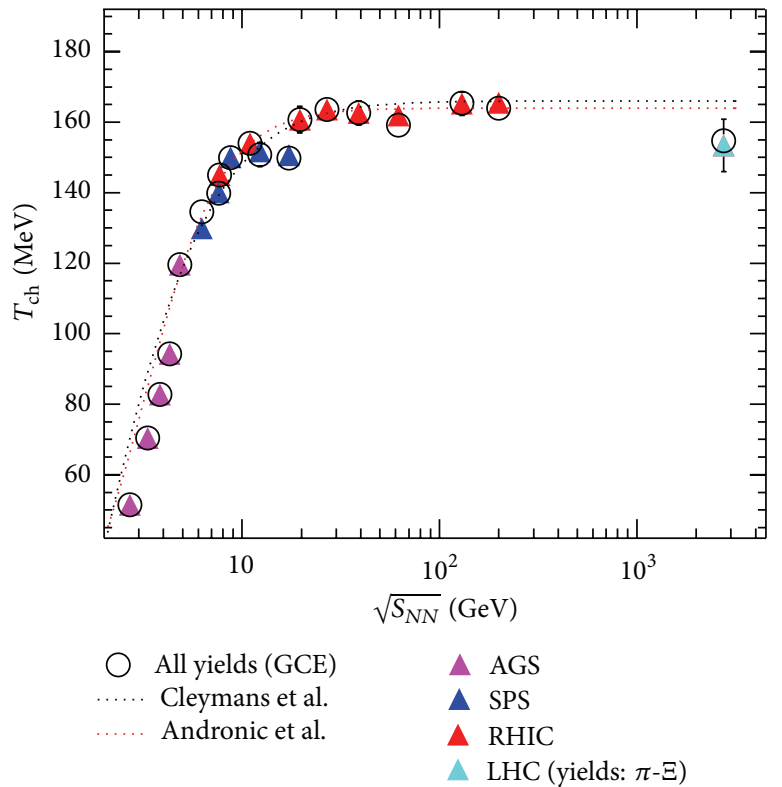

(a)

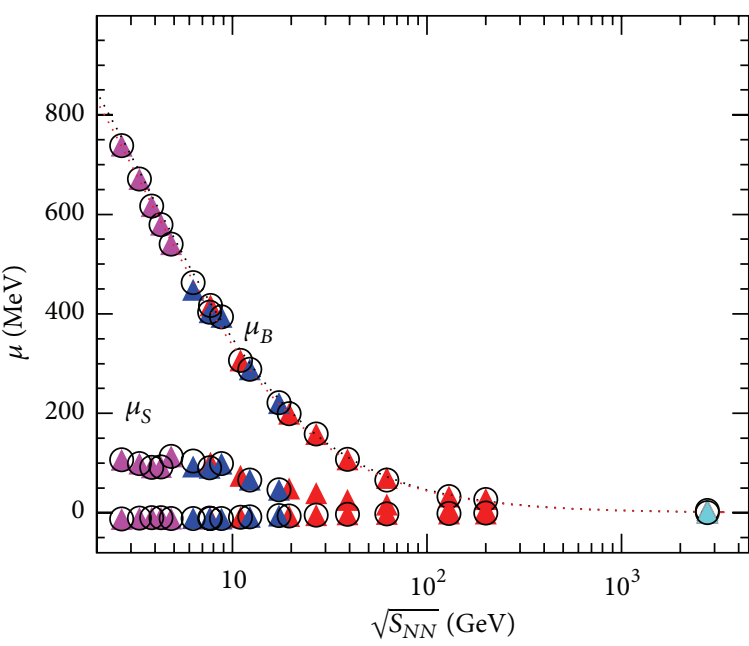

$\bigcirc$ All yields (GCE) Cleymans et al. Andronic et al.
$\triangle$ AGS
A SPS
$\triangle$ RHIC
$\triangle$ LHC (yields: $\pi-\Xi$ )

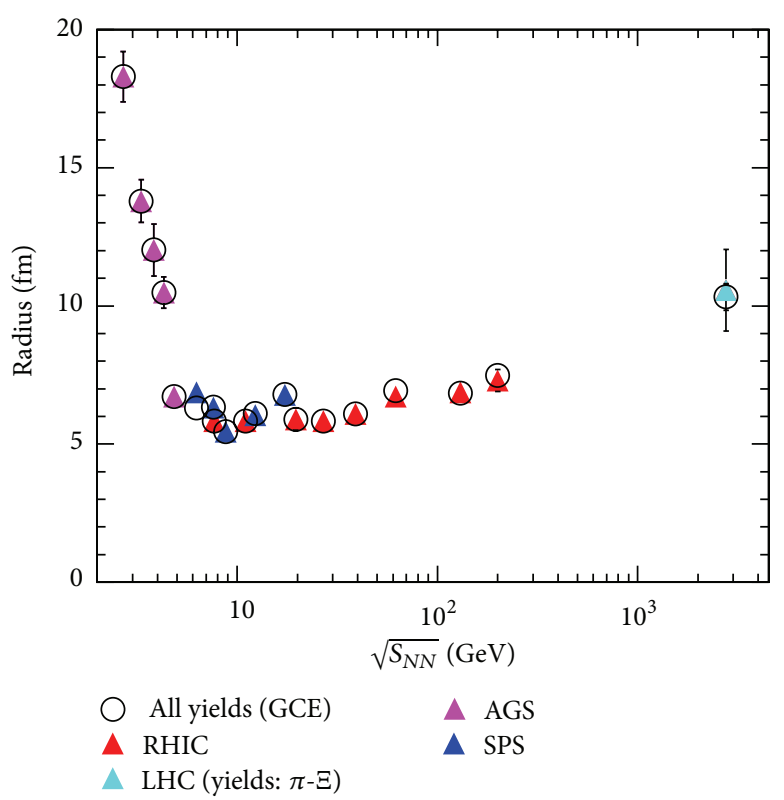

(c)

Figure 3: Dependence on the Choice of Particles Whose Yields Are Used as Input to Extract the Thermal Parameters. Comparative study of the thermal parameters (a) $T_{\mathrm{ch}}$, (b) $\mu\left(\mu_{\mathrm{B}}, \mu_{\mathrm{S}}\right.$, and $\left.\mu_{\mathrm{Q}}\right)$, and (c) radius, plotted versus collision energy, extracted within GCE with 1CFO as the freeze-out scheme for the two cases: (i) $\pi^{+}, \pi^{-}, K^{+}, K^{-}, p, \bar{p}, \Lambda, \bar{\Lambda}, \Xi$, and $\bar{\Xi}$ were available, and (ii) all available particles at all energies as tabulated in Tables 1, 2, 3, and 4. Here, $\gamma_{s}=1, \mu_{S}$ fixed from (2) and $\mu_{\mathrm{O}}$ fixed from (1). The fitted parameters are broadly insensitive to the choice of particles. However, at the AGS energies, because of the unavailability of data of the strange baryons, the extracted values of $\mu_{S}$ and $\mu_{\mathrm{Q}}$ come out to be much lower than expected from the trend at the SPS and higher energies.

obtained corresponding to a given energy using relation as in [81]. For a given $T_{\mathrm{ch}}$ and $\mu_{B}$, one can predict nuclei yields and hence ratios at a given energy. As discussed above, the $d / p$ being a like flavor ratio does not discriminate between the different freeze-out schemes (1CFO or 2CFO) while $\frac{3}{\Lambda} \bar{H} /{ }^{3} \overline{\mathrm{He}}$ being representing strange to nonstrange ratio shows the difference between two schemes. For the latter, the well known discrepancy between data and thermal model with $1 \mathrm{CFO}$ at $\sqrt{s_{N N}}=200 \mathrm{GeV}[85,86]$ goes away in $2 \mathrm{CFO}$ [26]. The grand canonical approach in THERMUS explains 


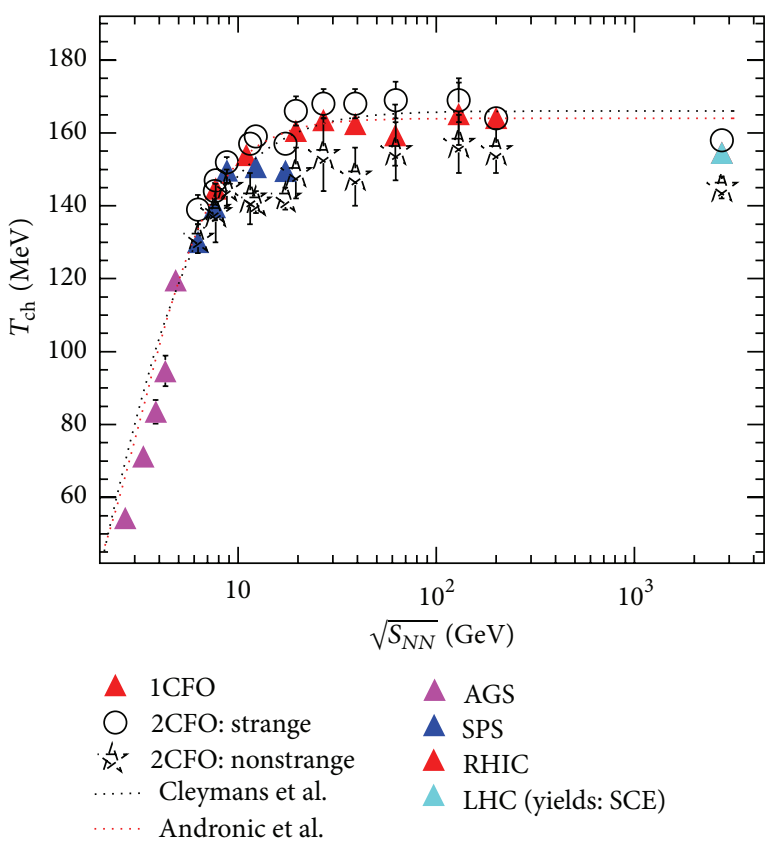

(a)

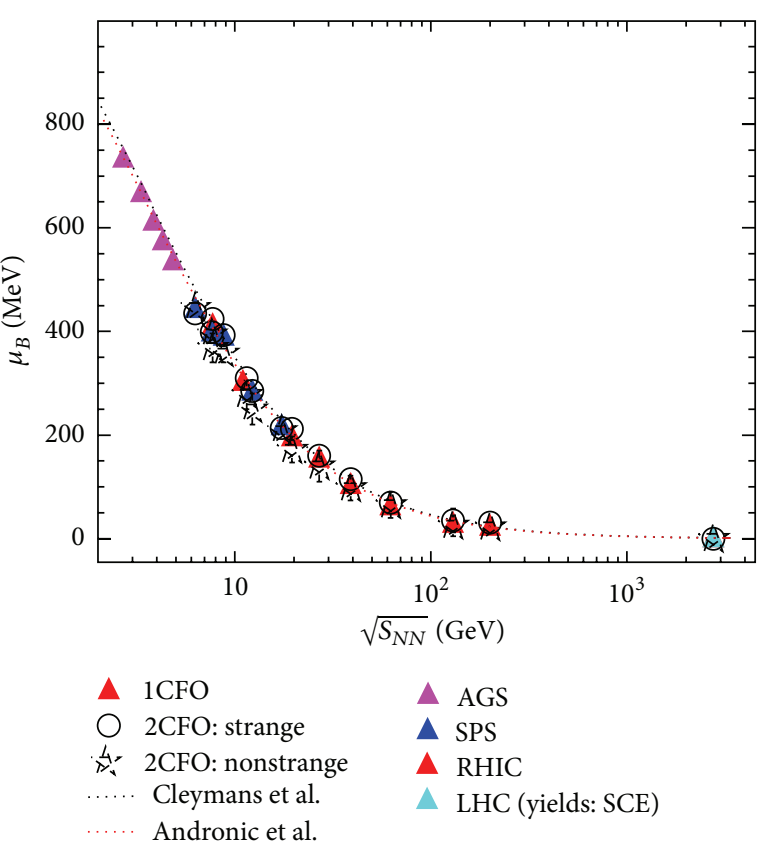

(b)

Figure 4: Dependence on the Choice of CFO Scheme. Comparative study of the thermal parameters (a) $T_{\mathrm{ch}}$ and (b) $\mu_{B}$, plotted versus collision energy, extracted within SCE with 1 CFO and 2 CFO as the freeze-out schemes with $\gamma_{s}=1$ and $\mu_{\mathrm{Q}}$ obtained by solving (1). The 1CFO parameters values are flanked by the $2 \mathrm{CFO}$ values for the nonstrange and strange surfaces.

the $d / p$ ratio very well; however, $\frac{3}{\Lambda} \bar{H} / 3 \overline{H e}$ ratio at $\sqrt{s_{N N}}=$ $200 \mathrm{GeV}$ is quite high in data compared to the expected value in THERMUS for both the GCE as well as the SCE approach.

\section{Transverse Energy per Charged Particle and Freeze-Out Criteria}

The transverse energy is one of the important global observables used to characterize the system formed in heavy-ion collisions at extreme conditions of temperature and energy density, where the formation of QGP is expected. The transverse energy $\left(E_{T}\right)$ is the energy produced transverse to the beam direction and is closely related to the collision geometry. $E_{T}$ is an event-by-event variable defined as

$$
E_{T}=\sum_{i} E_{i} \sin \theta_{i}, \quad \frac{d E_{T}(\eta)}{d \eta}=\frac{\sin \theta(\eta) d E(\eta)}{d \eta} .
$$

The sum is taken over all particles produced in an event into a fixed but large solid angle. $E_{i}$ and $\theta_{i}$ are the energy and polar angle that a final state particle makes with the detector. The energy of the individual particles can be measured by knowing their momenta and particle identification and/or the total energy deposited in a calorimeter. To probe the early stages of the produced fireball, it is ideal to take transverse observables such as $E_{T}$ and $p_{T}$. This is because, before the collision of two nuclei, the longitudinal phase space is filled by the beam particles whereas the transverse phase space is empty. The $E_{T}$ is produced due to the initial scattering of the partonic constituents of the incoming nuclei and also by the rescattering among the produced partons and hadrons $[87,88]$. The $E_{T}$ production tells about the explosiveness of the interaction. In addition, the collision centrality can be estimated by using the $E_{T}$ distribution [89]. The ratio of pseudorapidity densities of transverse energy and number of charged particles at midrapidity, that is, $\left(d E_{T} / d \eta\right) /\left(d N_{\mathrm{ch}} / d \eta\right)\left(\equiv E_{\mathrm{T}} / N_{\mathrm{ch}}\right)$, has been studied both experimentally $[65,69,90]$ and phenomenologically [91-93] to understand the underlying particle production mechanism. This observable is known as global barometric measure of the internal pressure in the ultradense matter produced in heavy-ion collisions [94]. This quantity depends on the initial state of the collision and the viscosity of the matter as it expands to its final state, when it is observed by the detectors [95].

This observable when studied as a function of collision energy (as shown in Figure 8), shows three regions of interest. The first one from the lower SIS energies to SPS energies shows a steep increase of $E_{T} / N_{\mathrm{ch}}$ values, thereby indicating that the mean energy of the system increases. In the second region, from SPS to top RHIC energy, $E_{T} / N_{\text {ch }}$ shows a very weak collision energy dependence, that is, like a saturation behaviour (Table 5 and Figure 8). In this region the mean energy does not increase, whereas the collision energy increases. This may indicate that the increase in collision energy results in new particle production in this energy domain, which is consistent with higher particle multiplicity observed at these energies. This behaviour has been well described in the context of a Statistical Hadron Gas Model (SHGM) [91, 92]. In the framework of SHGM, it has been 

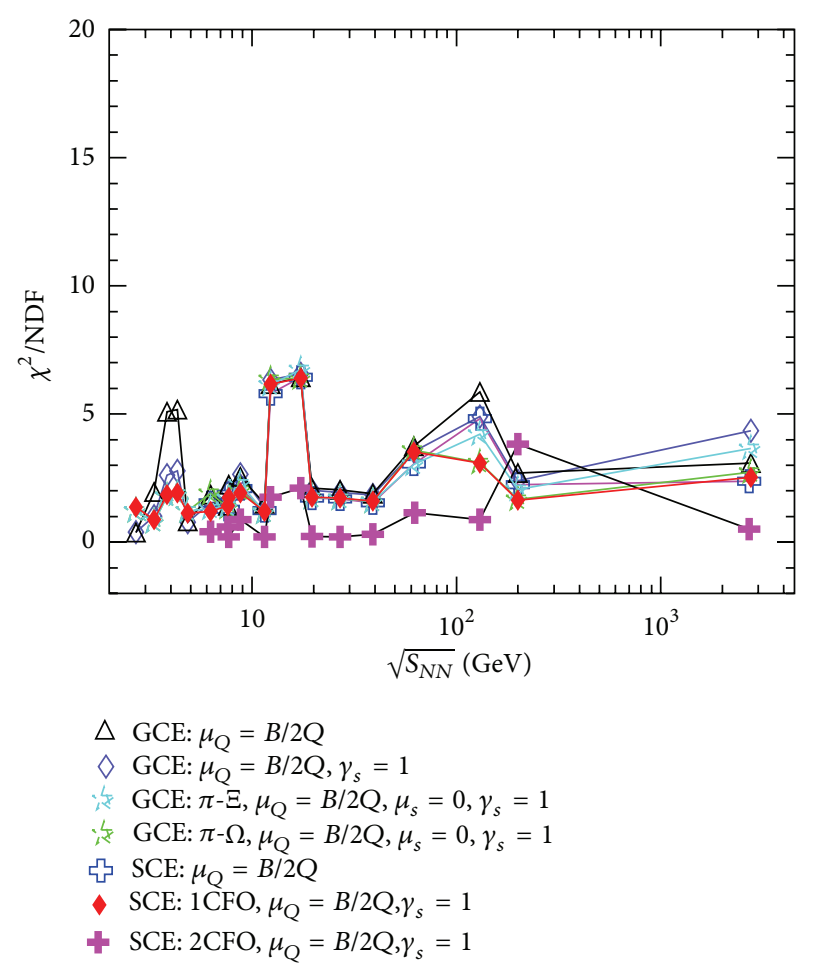

FIGURE 5: The $\chi^{2} / \mathrm{NDF}$ for the different cases in GCE and SCE along with $1 \mathrm{CFO}$ and $2 \mathrm{CFO}$ schemes are shown together for comparison.

predicted that $E_{T} / N_{\mathrm{ch}}$ would saturate at energies higher to that of top RHIC energy with a limiting value of $0.83 \mathrm{GeV}$ $[91,92]$. Here, a static fireball is assumed at the freeze-out. However, a value of $1.25 \pm 0.08 \mathrm{GeV}$ is observed at the LHC $2.76 \mathrm{TeV}$ center of mass energy recently, by the CMS collaboration [69]. This creates a third region in the excitation function of $E_{T} / N_{\mathrm{ch}}$, showing a jump from top RHIC to LHC energies. In this region, along with particle multiplicity, the mean energy per particle also increases, which needs to be understood from theoretical models taking the dynamics of the time evolution of the created fireball. It is however, observed that models based on final state gluon saturation (CGC like) seem to explain this behaviour in the excitation function of $E_{T} / N_{\mathrm{ch}}$ [96]. The RHIC BES data seem to follow the overall trend of the collision energy dependence of $E_{T} / N_{\mathrm{ch}}$. It has been seen in one of the previous works [92] that various freeze-out criteria like constant energy per particle $(\langle E\rangle /\langle N\rangle=1.08 \mathrm{GeV})$ [97], fixed baryon + anti-baryon density $\left(n_{B}+n_{\bar{B}} \simeq 0.12 \mathrm{fm}^{-3}\right)$ [98], fixed entropy density per $T^{3}\left(s / T^{3} \simeq 7\right)[79,99]$ seem to describe the qualitative energy dependent behaviour of $E_{T} / N_{\text {ch }}$ quite consistently.

The behavior $\left(d E_{T} / d \eta\right) /\left(d N_{\mathrm{ch}} / d \eta\right)\left(\equiv E_{T} / N_{\mathrm{ch}}\right)$ as a function of energy can naively be related to first order phase transition as discussed below. If we assume the system to be following thermodynamic behavior, then $E_{T} / N_{\text {ch }}$ may be interpreted as temperature at freeze-out (volume considered to be constant at freeze-out) while the collision energy may be interpreted as entropy. This figure, then could be considered as temperature versus entropy diagram, where an observed
TABLE 5: $E_{T} / N_{\text {ch }}$ as a function of $\sqrt{s_{N N}}$, plotted in Figure 8.

\begin{tabular}{lccc}
\hline$\sqrt{s_{N N}}(\mathrm{GeV})$ & Coll. species & $E_{T} / N_{\text {ch }}(\mathrm{GeV})$ & Reference \\
\hline 2.05 & $\mathrm{Au}+\mathrm{Au}$ & $0.13 \pm 0.03$ & {$[64]$} \\
3.81 & $\mathrm{Au}+\mathrm{Au}$ & $0.598 \pm 0.060$ & {$[65]$} \\
4.27 & $\mathrm{Au}+\mathrm{Au}$ & $0.634 \pm 0.063$ & {$[65]$} \\
4.84 & $\mathrm{Au}+\mathrm{Au}$ & $0.680 \pm 0.068$ & {$[65]$} \\
8.7 & $\mathrm{~Pb}+\mathrm{Pb}$ & $0.760 \pm 0.060$ & {$[42,66]$} \\
12.4 & $\mathrm{~Pb}+\mathrm{Pb}$ & $0.780 \pm 0.060$ & {$[42,66]$} \\
17.2 & $\mathrm{~Pb}+\mathrm{Pb}$ & $0.810 \pm 0.060$ & {$[67]$} \\
19.6 & $\mathrm{Au}+\mathrm{Au}$ & $0.738 \pm 0.070$ & {$[65]$} \\
62.4 & $\mathrm{Au}+\mathrm{Au}$ & $0.867 \pm 0.121$ & {$[68]$} \\
130 & $\mathrm{Au}+\mathrm{Au}$ & $0.869 \pm 0.066$ & {$[65]$} \\
200 & $\mathrm{Au}+\mathrm{Au}$ & $0.881 \pm 0.071$ & {$[65]$} \\
2760 & $\mathrm{~Pb}+\mathrm{Pb}$ & $1.283 \pm 0.085$ & {$[69]$} \\
\hline
\end{tabular}

plateau represents a first order phase transition [100]. Therefore, one can say that temperature increases at very lower energies with an increase of collision energy, then remains almost constant from collision energy of about $20 \mathrm{GeV}$ up to the top RHIC energies. Then, it shows an increase again from top RHIC to LHC energies. This observation of constant temperature from 20 to $200 \mathrm{GeV}$ can be interpreted as the first order phase transition turning on around $\sqrt{s_{N N}}=20 \mathrm{GeV}$. Similar behavior is observed for the $\left\langle m_{T}\right\rangle-m$ versus collision energy, $m_{T}$ being the transverse mass [53].

Figure 9 shows the $E_{T} / N_{\text {ch }}$ plotted versus $\left\langle N_{\text {part }}\right\rangle$, which is a measure of centrality. Table 6 shows the corresponding $E_{T} / N_{\text {ch }}$ values for different centralities at various energies. Within the systematic errors, $E_{T} / N_{\mathrm{ch}}$ for all energies show a weak centrality dependence with a modest increase from most peripheral collisions to $\left\langle N_{\text {part }}\right\rangle=100$, reaching a roughly constant value towards central collisions. This centrality dependence of $E_{T} / N_{\mathrm{ch}}$ is shown to be equivalent to the behaviour of $\left\langle p_{T}\right\rangle$ as a function of centrality for top RHIC energy [90] and for $\sqrt{s_{N N}}=2.76 \mathrm{TeV}$ [101] at LHC. The value of $\left\langle p_{T}\right\rangle=0.685 \pm 0.016 \mathrm{GeV} / \mathrm{c}$ for $\sqrt{s_{N N}}=2.76 \mathrm{TeV}$, which is almost $37 \%$ higher as compared to the $\left\langle p_{T}\right\rangle(\sim 0.5 \mathrm{GeV} / \mathrm{c})$ at top RHIC energy [90]. This shows that not only particle multiplicity increases while going from top RHIC energy to LHC energy, the $\left\langle p_{T}\right\rangle$ also increases, making a third region in the excitation function of $E_{T} / N_{\mathrm{ch}}$. The near centrality independent behaviour of $E_{T} / N_{\mathrm{ch}}$ is explained by SHGM with a static fireball approximation at freeze-out [91]. If the freeze-out is assumed to occur at all collision energies and impact parameters in heavy-ion collisions on a fixed decoupling isotherm, then the energy per particle will always be the same. This possibly is the fact up to top RHIC energy within experimental uncertainties. However, the LHC measure of a higher value of $E_{T} / N_{\mathrm{ch}}$ needs to be understood in the above context keeping in mind that there is hardly any change of $T_{\mathrm{ch}}$ while going from top RHIC energy to LHC energy at $2.76 \mathrm{TeV}$ [102]

At higher energies, when $\mu_{B} \sim 0$, the transverse energy production is mainly due to the meson content of the system. The experimental observations go in line with the above fact, when we observe the ratio of $\bar{p} / p \sim 1$ at higher energies. The 


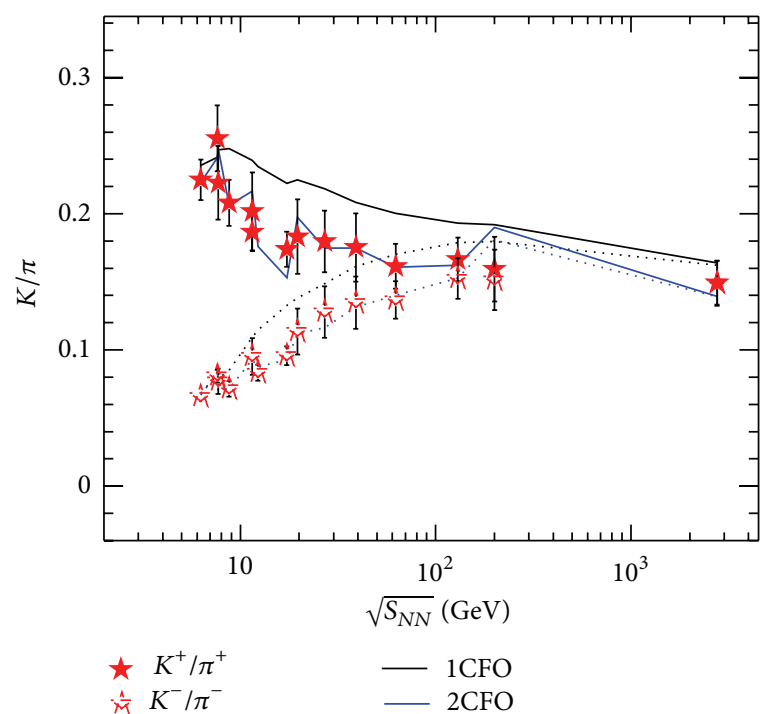

(a)

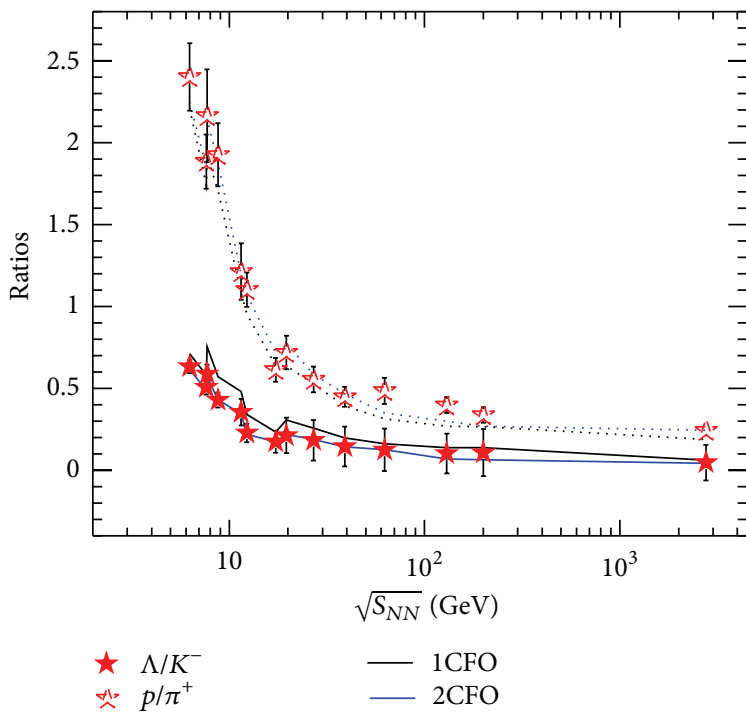

(c)

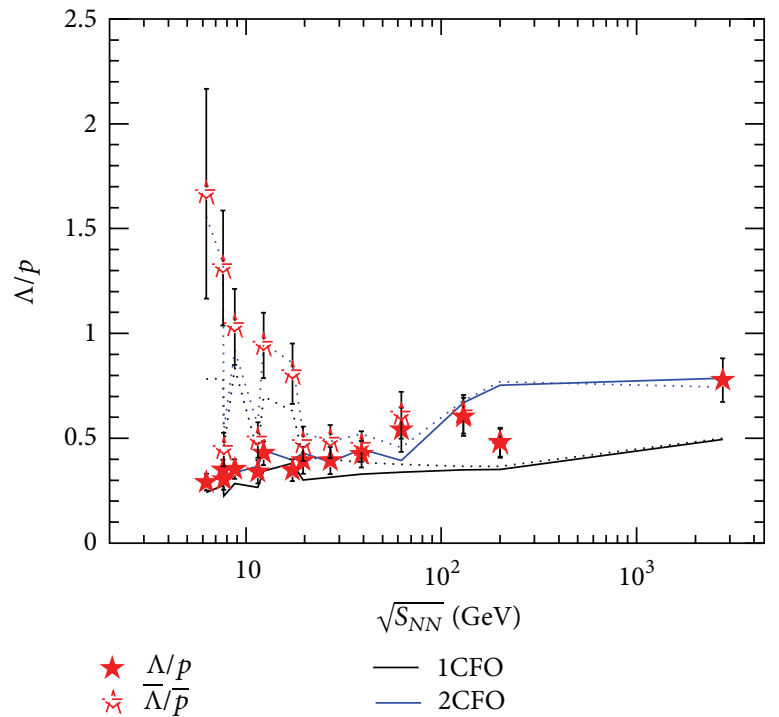

(b)

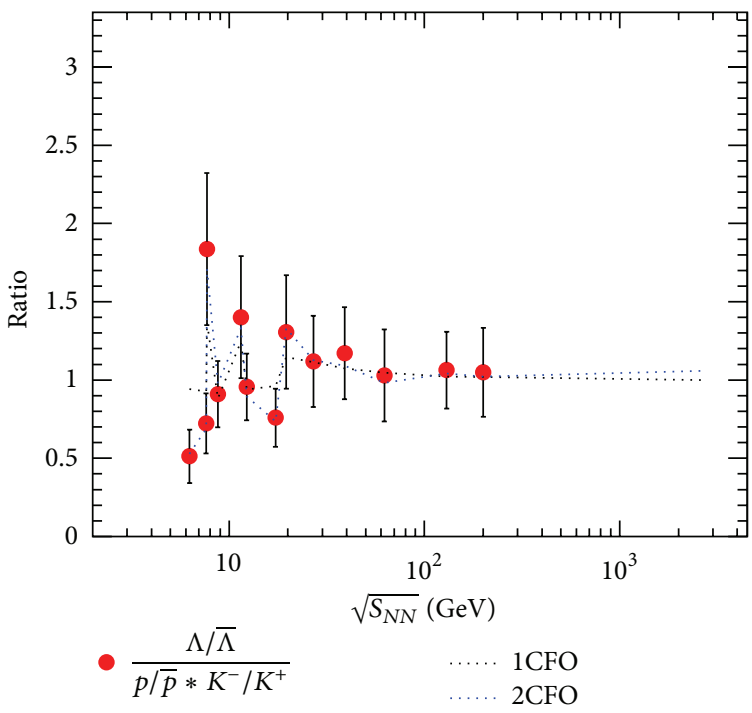

(d)

Figure 6: Dependence on the Choice of CFO Scheme on Several Particle Ratios. Comparative study of different particle ratios (a) $K^{ \pm} / \pi^{ \pm}$, (b) $\Lambda / p(\bar{\Lambda} / \bar{p})$, (c) $\Lambda / K^{-}$and $p / \pi^{+}$, and (d) triple ratio $\left((\Lambda / \bar{\Lambda}) /\left((p / \bar{p})\left(K^{-} / K^{+}\right)\right)\right)$, plotted versus collision energy, within SCE with $1 \mathrm{CFO}$ and $2 \mathrm{CFO}$ as the freeze-out schemes with $\gamma_{s}=1$. The solid curves in the figures (a), (b), and (c) represent 1CFO (black curve) and 2CFO (blue curve) schemes corresponding to ratios shown with solid symbols. The corresponding dotted curves for 1CFO (black) and 2CFO (blue) are for the ratios shown with open symbols.

TABLE 6: $E_{T} / N_{\text {ch }}$ for different centralities corresponding to the $\left\langle N_{\text {part }}\right\rangle$ values as shown in Figure 9 for various energies.

\begin{tabular}{|c|c|c|c|c|c|c|c|c|c|c|c|c|c|c|}
\hline $\begin{array}{l}\sqrt{s_{N N}} \\
(\mathrm{GeV})\end{array}$ & $0-5 \%$ & $5-10 \%$ & $10-15 \%$ & $15-20 \%$ & $20-25 \%$ & $25-30 \%$ & $30-35 \%$ & $35-40 \%$ & $40-45 \%$ & $45-50 \%$ & $50-55 \%$ & $55-60 \%$ & $60-65 \%$ & $65-70 \%$ \\
\hline 7.7 & 0.70 & 0.73 & 0.74 & 0.77 & 0.78 & 0.81 & 0.84 & 0.87 & 0.90 & 0.94 & & & & \\
\hline 19.6 & 1.07 & 1.11 & 1.14 & 1.17 & 1.18 & 1.22 & 1.24 & 1.27 & 1.30 & 1.36 & & & & \\
\hline 27 & 1.22 & 1.24 & 1.27 & 1.30 & 1.34 & 1.38 & 1.40 & 1.44 & 1.47 & 1.54 & & & & \\
\hline 39 & 1.41 & 1.45 & 1.47 & 1.51 & 1.54 & 1.58 & 1.61 & 1.66 & 1.71 & 1.69 & & & & \\
\hline 62.4 & 1.21 & 1.26 & 1.32 & 1.40 & 1.49 & 1.57 & 1.67 & 1.76 & 1.84 & 1.92 & 2.01 & 2.07 & 2.17 & 2.23 \\
\hline 130 & 1.95 & 2.02 & 2.05 & 2.12 & 2.21 & 2.31 & 2.38 & 2.48 & 2.56 & 2.65 & 2.73 & 2.80 & 2.89 & 3.01 \\
\hline 200 & 1.93 & 2.06 & 2.18 & 2.31 & 2.46 & 2.57 & 2.66 & 2.76 & 2.87 & 2.95 & 3.04 & 3.16 & 3.29 & 3.44 \\
\hline
\end{tabular}




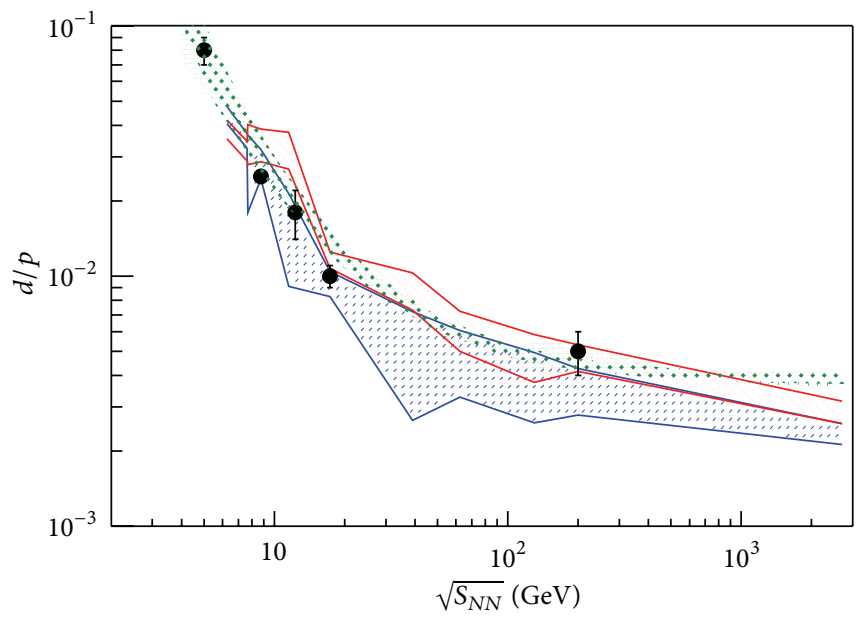

GCE (thermus)

(a)

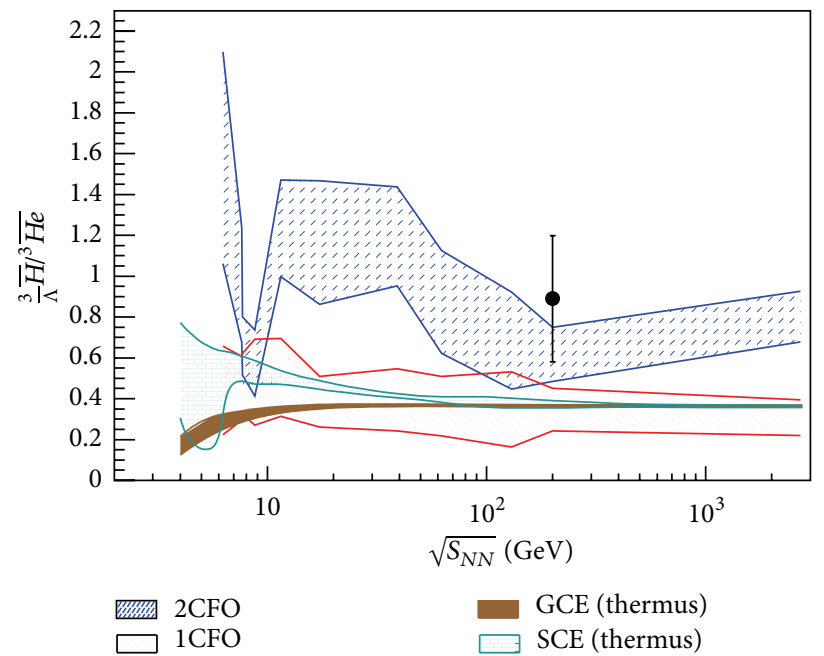

(b)

Figure 7: Comparison of light nuclei experimental data ratios (a) $d / p$ and (b) $\frac{3}{\Lambda} \bar{H} / 3 \overline{H e}$, plotted versus collision energy, with 1CFO and 2CFO as the CFO schemes along with THERMUS model expectations, also representing $1 \mathrm{CFO}$.

intersection points of lines of constant $E_{T} / N_{\mathrm{ch}}$ and the freezeout line give the values of $E_{T} / N_{\mathrm{ch}}$ at the chemical freeze-out.

\section{Kinetic Freeze-Out}

At chemical freeze-out, the inelastic interactions among the produced particles stop. However, these particles can still interact elastically which could affect their momentum distributions. A point in time after the collision, when the elastic interactions among the particles also stop is called the kinetic freeze-out. At that time, the transverse momentum distributions of the particles get fixed and do not change thereafter. The transverse momentum distribution of particles contains two components-random and the collective. The random component depends on the temperature of the system at kinetic freeze-out $\left(T_{\text {kin }}\right)$. The collective component is generated by collective flow in the transverse direction given by the transverse flow velocity $\beta$.

The $T_{\text {kin }}$ and average transverse flow velocity $\langle\beta\rangle$ of the system can be obtained using the hydrodynamics-motivated Blast Wave model [6-11], assuming thermal equilibrium. The model assumes that the particles are locally thermalized at kinetic freeze-out temperature and are moving with a common transverse collective flow velocity. Assuming a radially boosted thermal source, with a kinetic freeze-out temperature $T_{\text {kin }}$ and transverse radial flow velocity $\beta$, the transverse momentum $p_{T}$ distribution of the particles can be given by

$$
\begin{aligned}
\frac{d N}{p_{T} d p_{T}} \propto & \int_{0}^{R} r d r m_{T} I_{0}\left(\frac{p_{T} \sinh \rho(r)}{T_{\text {kin }}}\right) \\
& \times K_{1}\left(\frac{m_{T} \cosh \rho(r)}{T_{\text {kin }}}\right),
\end{aligned}
$$




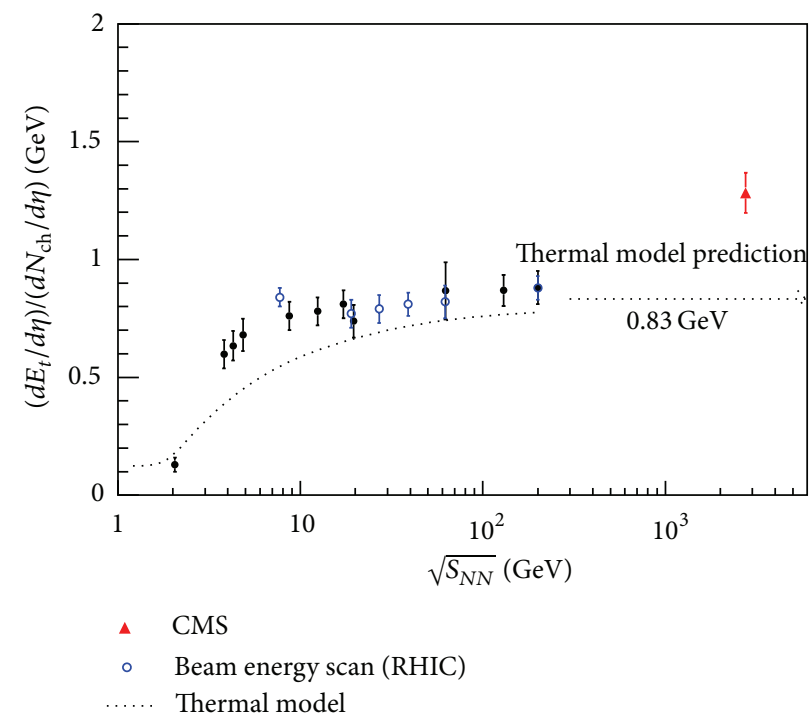

Figure 8: The midrapidity $E_{T} / N_{\mathrm{ch}}$ as a function of collision energy for few $\mathrm{GeV}$ to $\mathrm{TeV}$ energies for the most central collisions.

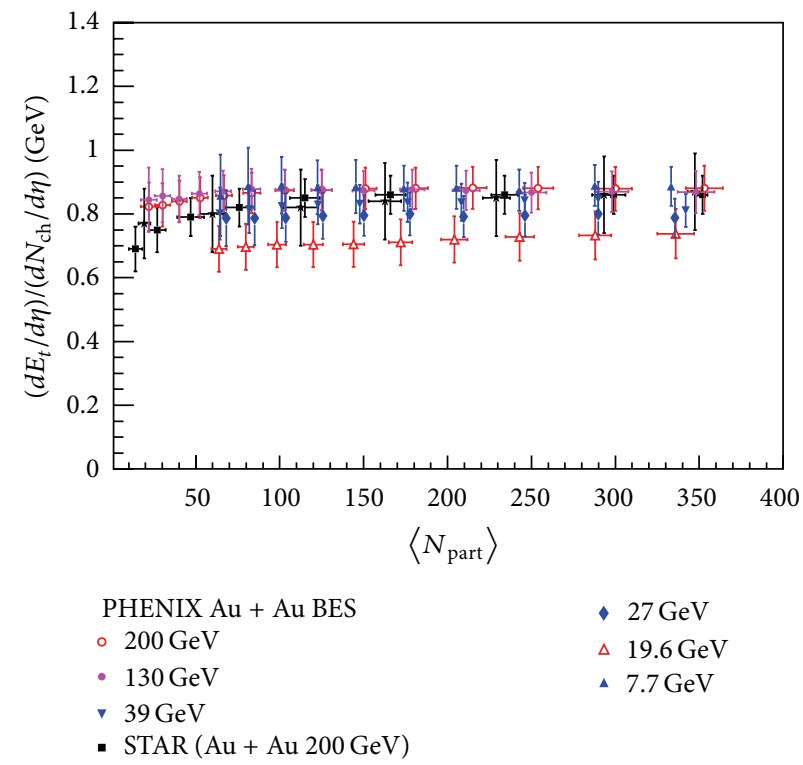

FIGURE 9: The midrapidity $E_{T} / N_{\mathrm{ch}}$ as a function of collision centrality for a wide range of energies spanning RHIC Beam energy scan to LHC.

where $m_{T}=\sqrt{p_{T}^{2}+m^{2}}, \rho(r)=\tanh ^{-1} \beta$, and $I_{0}$ and $K_{0}$ are the modified Bessel functions. We use the flow velocity profile of the form $\beta=\beta_{S}(r / R)^{n}$, where $\beta_{S}$ is the surface velocity, $r / R$ is the relative radial position in the thermal source, and $n$ is the exponent of flow velocity profile. Average transverse radial flow velocity $\langle\beta\rangle$ can then be obtained as $\langle\beta\rangle=(2 /(2+$ n)) $\beta_{S}$.

Usually, the $p_{T}$ spectra of the six particles $\left(\pi^{ \pm}, K^{ \pm}, p\right.$ and $\bar{p}$ ) are fitted simultaneously with the blast wave model to extract the parameters such as $T_{\text {kin }},\left\langle\beta_{T}\right\rangle$, and $n$. Figure 10 shows the $p_{T}$ spectra for $\pi^{-}, K^{-}$, and $\bar{p}$ in central collisions at different energies. For clarity, the $p_{T}$ spectra for positively charged particles are not shown. These spectra are used to extract the kinetic freeze-out parameters using blast wave model. The curves in the figure represent the blast wave model fits to the data and can be seen to reasonably describe the $p_{T}$ spectra of $\pi, K, p$, and $\bar{p}$ at all energies. The results compiled here are taken from $[42,53,55,70-72]$. All these results use same particles $\left(\pi^{ \pm}, K^{ \pm}, p\right.$ and $\left.\bar{p}\right)$ for blastwave fitting except the $10.8 \mathrm{~A} \mathrm{GeV}\left(\sqrt{s_{N N}}=4.8 \mathrm{GeV}\right)$ energy corresponding to AGS, which use $\left(\pi^{+}+\pi^{-}\right) / 2, K^{+}, p$, and $d$ spectra; and $\sqrt{s_{N N}}=17.3 \mathrm{GeV}$, where $\pi^{-}, K^{ \pm}, p$, and $\bar{p}$ are used. The $p_{T}$ ranges used for fitting of these spectra are given in Table 7. The low $p_{T}$ part of pion spectra is affected by resonance decays due to which the pion spectra is fitted above $p_{T}>0.5 \mathrm{GeV} / \mathrm{c}$ or $\left(m_{T}-m\right)>0.4 \mathrm{GeV} / \mathrm{c}^{2}$ for $\sqrt{s_{N N}}=4.8 \mathrm{GeV}$.

Figure 11(a) shows the energy dependence of kinetic and chemical freeze-out temperatures and Figure 11(b) shows $T_{\mathrm{ch}}$ 


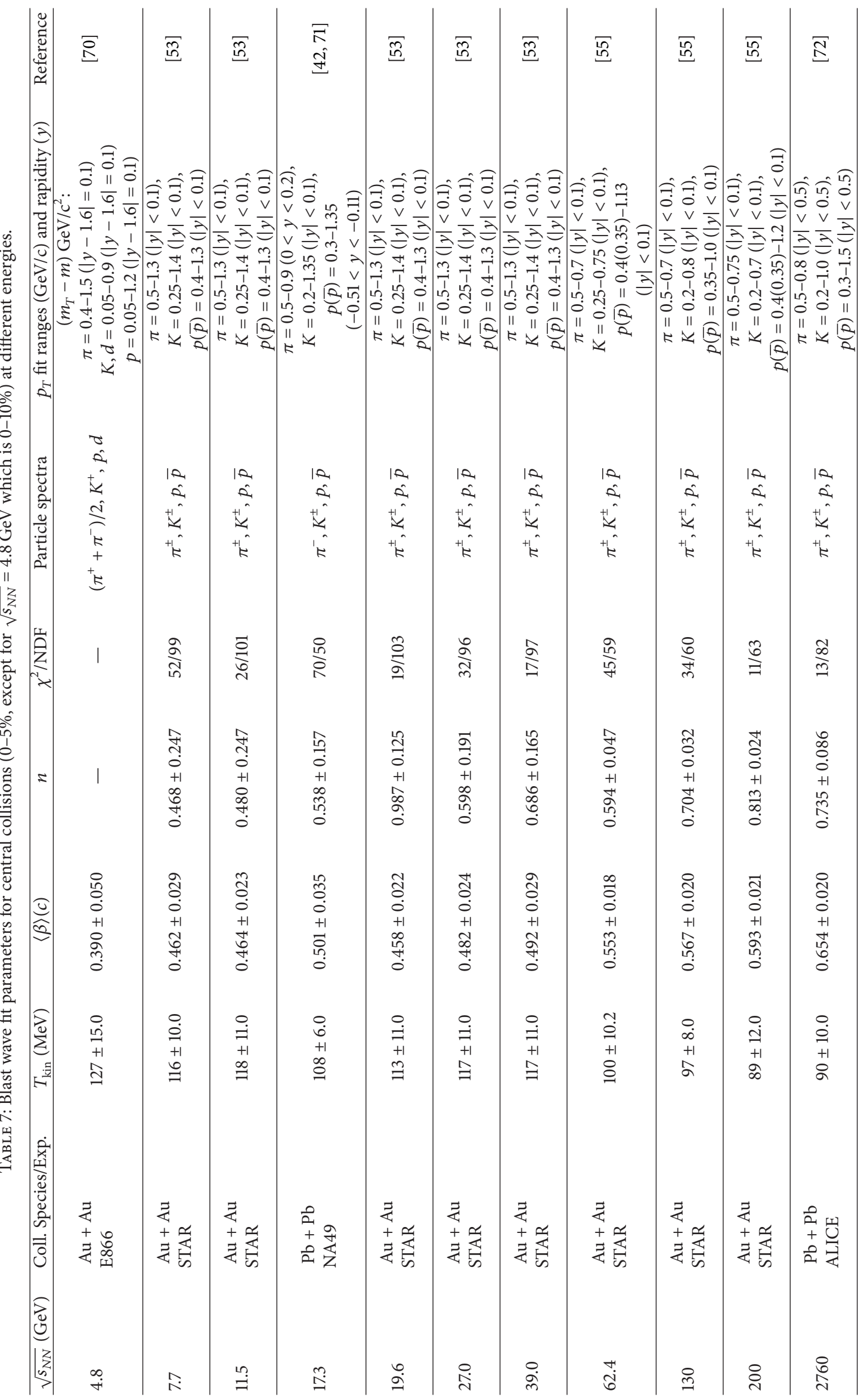




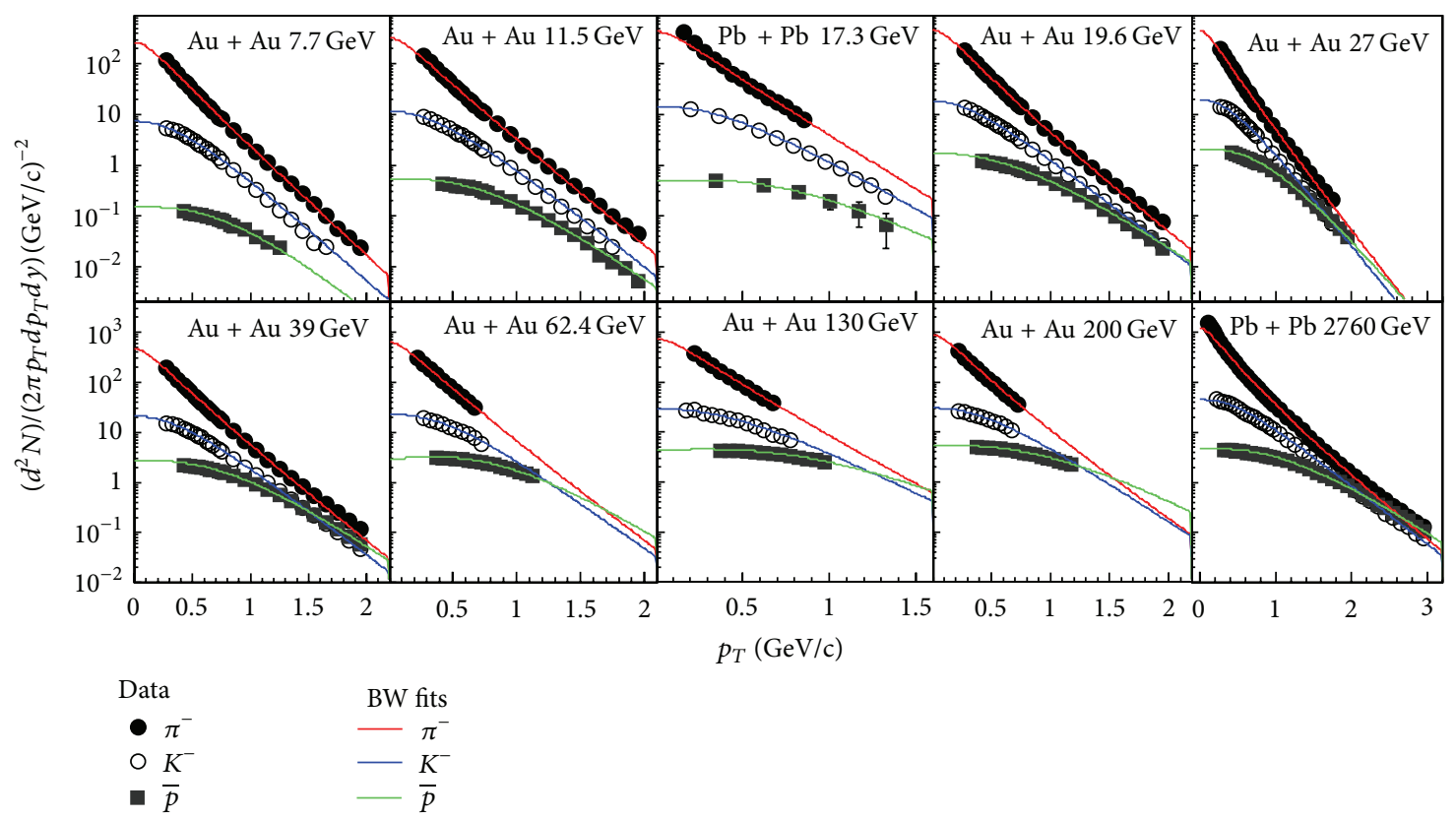

Figure 10: (Color online) invariant yields of $\pi^{-}, K^{-}$, and $\bar{p}$ versus $p_{T}$ in central collisions at different energies. The curves represent the blast wave fits. For more details about centralities, rapidities, and $p_{T}$ fit ranges, please refer to Table 7 .

and $T_{\text {kin }}$ plotted versus $\mu_{B}$. For chemical freeze-out temperature and/or $\mu_{B}$, we have taken the values obtained using $1 \mathrm{CFO}$ GCE fit to the particle yields as discussed in previous sections. We observe that the values of kinetic and chemical freeze-out temperatures are similar around $\sqrt{s_{N N}}=4-5 \mathrm{GeV}$. If the collision energy is increased, the chemical freezeout temperature increases and becomes constant after the $\sqrt{s_{N N}}=11.5 \mathrm{GeV}$. However, the $T_{\text {kin }}$ is almost constant around the $7.7-39 \mathrm{GeV}$ and then decreases up to LHC energies. The separation between $T_{\mathrm{ch}}$ and $T_{\text {kin }}$ increases with increasing energy (or decreasing $\mu_{B}$ ). This might suggest the effect of increasing hadronic interactions between chemical and kinetic freeze-out towards higher energies.

Figure 12 shows the average flow velocity plotted as a function of $\mu_{B}$. Value of $\langle\beta\rangle$ is about 0.4 times the speed of light at around $\mu_{B}=500 \mathrm{MeV}$. For $\mu_{B}=200-400 \mathrm{MeV}$, the $\langle\beta\rangle$ remains almost constant and then increases when the $\mu_{B}$ decreases to lower values corresponding to LHC energies. The SPS (NA49) data at $\mu_{B} \approx 220 \mathrm{MeV}$ (corresponding to $\sqrt{s_{N N}}=$ $17.3 \mathrm{GeV}$ ) has mean value little higher although consistent within error bars with BES results at nearby energies. This slight difference between BES and SPS data is may be due to different particle species and rapidity regions (as given in Table 7) used in blast wave fitting. Since $\langle\beta\rangle$ reflects the expansion in the transverse direction, it is interesting to note that expansion is constant around $\mu_{B}=200-400 \mathrm{MeV}$ corresponding to beam energies $7.7,11.5$, and $19.6 \mathrm{GeV}$.

It may be noted that the blast-wave results are sensitive to the particle species as well as $p_{T}$-ranges and rapidity regions used for fits. However, it is difficult to satisfy consistent conditions for above mentioned properties across different experiments due to different technicality of detectors as well as due to different energy regimes where these experiments operate. In addition, the spectra also become harder and harder when we go towards higher energy. The results presented here though use common $p_{T}$ ranges but not exact same ranges as can be seen from Table 7. It will be interesting to see how the energy or $\mu_{B}$ dependence of $T_{\text {kin }}$ and $\langle\beta\rangle$ looks like if similar kinematic ranges and particle species are used. To demonstrate effect of $p_{T}$ ranges on the blast wave fitting, as an example, for ALICE experiment at LHC energy, we have tested to use same $p_{T}$ ranges as used in BES energies at RHIC (see Table 7). The mean values of the results obtained are $T_{\text {kin }}=104 \mathrm{MeV},\langle\beta\rangle=0.639$, and $n=0.710$. Similarly, using $p_{T}$ fit ranges: $0.5-1.0 \mathrm{GeV} / \mathrm{c}$ for pions, $0.2-1.5 \mathrm{GeV} / \mathrm{c}$ for kaons, and $0.3-3 \mathrm{GeV} / \mathrm{c}$ for protons, lead to the following mean values of fit parameters: $T_{\text {kin }}=95 \mathrm{MeV},\langle\beta\rangle=0.651$, and $n=0.712$ [72]. Clearly, the fit parameters are sensitive to fitting ranges and hence one needs to be careful interpreting results when kinematic conditions are different.

\section{Summary}

We have presented the systematic study of chemical and kinetic freeze-out conditions obtained at different energies from AGS, SPS, RHIC, and LHC.

The dependence of chemical freeze-out parameters on various factors like choice of thermodynamic ensemble, choice of free parameters and fitting procedures, choice of particles whose yields and/or particle ratios are used as inputs in the fits, and choice of chemical freeze-out schemes have been studied in detail. While we find weak dependence of the extracted thermodynamic parameters on most of the factors listed above, choice of chemical freeze-out scheme 


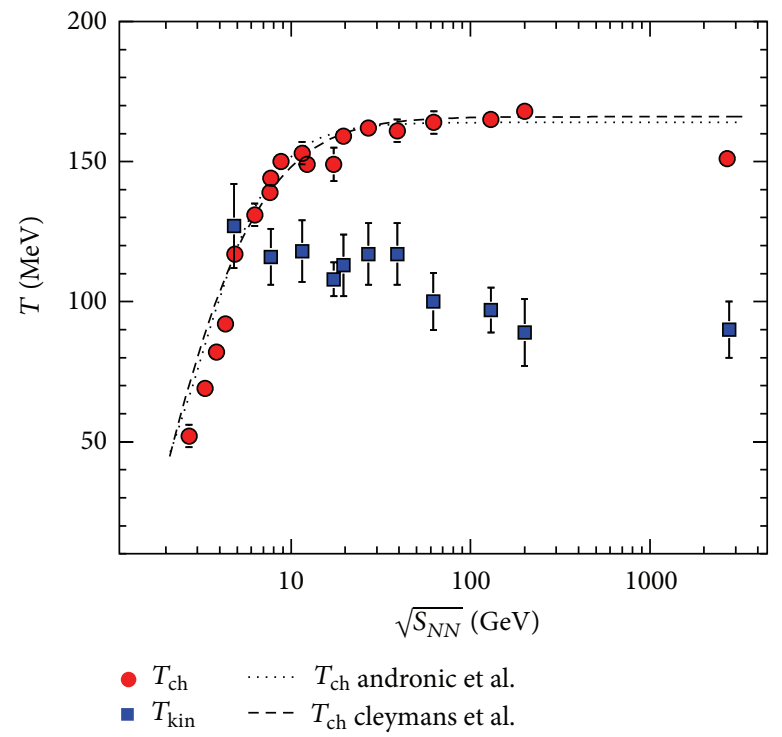

(a)

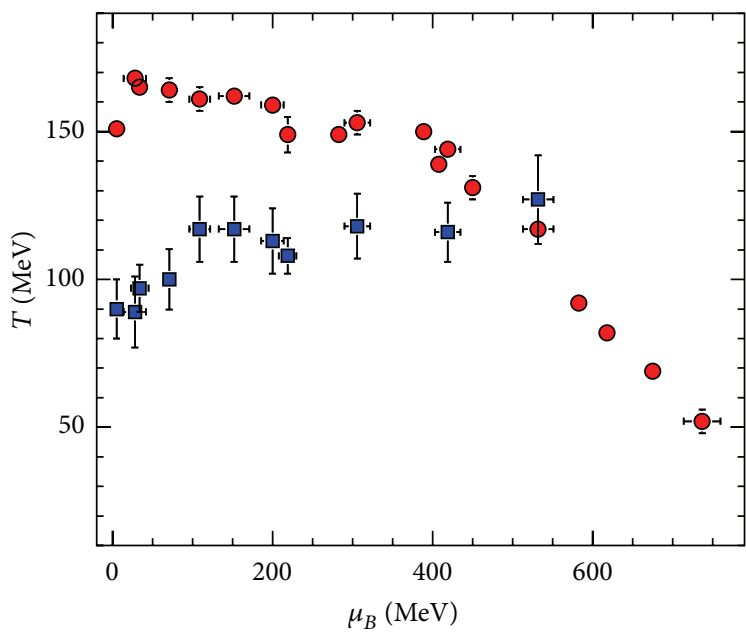

- Chemical FO

- Kinetic FO

(b)

Figure 11: (Color online) (a) energy dependence of $T_{\mathrm{ch}}$ and $T_{\text {kin }}$. Curves represent different theory predictions [81, 82]. (b) $T_{\mathrm{ch}}$ and $T_{\text {kin }}$ plotted versus $\mu_{B}$.

seems to influence the fitted thermal parameters significantly. Particularly, strange to nonstrange particle ratios are most sensitive to the chosen chemical freeze-out scheme.

The barometric observable, transverse energy per charged particle, is related to the chemical freeze-out. Various freezeout criteria seem to describe the energy dependent behavior of $E_{T} / N_{\text {ch }}$ starting from few $\mathrm{GeV}$ to top RHIC energies. A static fireball approximation at freeze-out, however, fails to reproduce the corresponding data at LHC and necessitates the inclusion of fireball evolution dynamics in space and time in order to describe the behavior for the whole range of energies. The similarity in the centrality dependence up to top RHIC energy indicates that irrespective of the collision species and center of mass energies, the system evolves to a similar final state at freeze-out.

The kinetic freeze-out temperature suggests a decrease from lower to higher energies. The separation between chemical and kinetic freeze-out temperatures increases while going towards lower $\mu_{B}$ (or higher energies) indicating increasing hadronic interactions between chemical and kinetic freezeout at higher energies. The average transverse radial flow velocity is around $40 \%$ of speed of light at $\mu_{B} \approx 500 \mathrm{MeV}$. The expansion in the radial direction remains similar around $\mu_{B}=$ 200-400 MeV and then increases towards lower $\mu_{B}$ or higher energies reflecting higher pressure or higher initial energy density produced in these collisions. 


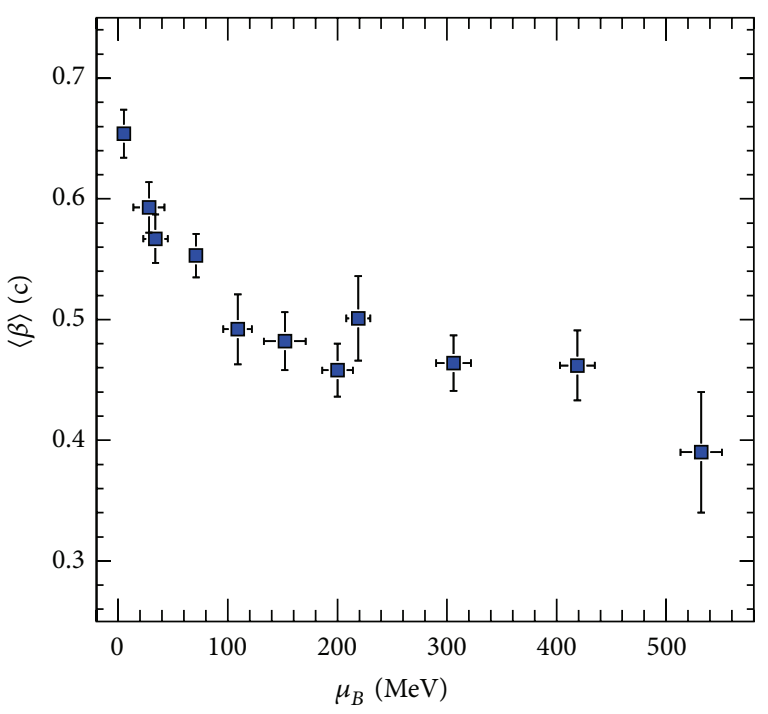

FIGURE 12: (Color online) $\langle\beta\rangle$ plotted as a function of $\mu_{B}$.

\section{Conflict of Interests}

The authors declare that there is no conflict of interests regarding the publication of this paper.

\section{Acknowledgment}

Bedangadas Mohanty is supported by the DAE-SRC project fellowship for this work.

\section{References}

[1] U. W. Heinz, "Concepts of heavy-ion physics," http://arxiv.org/ abs/hep-ph/0407360.

[2] P. Braun-Munzinger, J. Stachel, J. Wessels, and N. Xu, "Thermal and hadrochemical equilibration in nucleus-nucleus collisions at the SPS," Physics Letters B, vol. 365, pp. 1-6, 1996.

[3] G. D. Yen and M. I. Gorenstein, "The Analysis of particle multiplicities in $\mathrm{Pb}+\mathrm{Pb}$ collisions at CERN SPS within hadron gas models," Physical Review C, vol. 59, article 2788, 1999.

[4] F. Becattini, J. Cleymans, A. Keranen, E. Suhonen, and K. Redlich, "Features of particle multiplicities and strangeness production in central heavy ion collisions between 1.7A and 158A GeV/c," Physical Review C, vol. 64, Article ID 024901, 2001.

[5] A. Andronic, P. Braun-Munzinger, and J. Stachel, "Hadron production in central nucleus-nucleus collisions at chemical freeze-out," Nuclear Physics A, vol. 772, no. 3-4, pp. 167-199, 2006.

[6] E. Schnedermann, J. Sollfrank, and U. W. Heinz, "Thermal phenomenology of hadrons from $200 \mathrm{AGeV} \mathrm{S+S} \mathrm{collisions,"}$ Physical Review C, vol. 48, pp. 2462-2475, 1993.

[7] D. Teaney, J. Lauret, and V. E. Shuryak, "Flow at the SPS and RHIC as a quark gluon plasma signature," Physical Review Letters, vol. 86, p. 4783, 2001.

[8] P. Kolb, U. W. Heinz, P. Huovinen, K. Eskola, and K. Tuominen, "Centrality dependence of multiplicity, transverse energy, and elliptic flow from hydrodynamics," Nuclear Physics A, vol. 696, pp. 197-215, 2001.
[9] P. Huovinen, P. F. Kolb, U. W. Heinz, P. V. Ruuskanen, and S. A. Voloshin, "Radial and elliptic flow at RHIC: further predictions," Physics Letters B, vol. 503, no. 1-2, pp. 58-64, 2001.

[10] U. W. Heinz and P. F. Kolb, "Early thermalization at RHIC," Nuclear Physics A, vol. 702, pp. 269-280, 2002.

[11] F. Retière and M. A. Lisa, "Observable implications of geometrical and dynamical aspects of freeze-out in heavy ion collisions," Physical Review C, vol. 70, no. 4, Article ID 044907, 33 pages, 2004.

[12] A. Baran, W. Broniowski, and W. Florkowski, "Description of the particle ratios and transverse-momentum spectra for various centralities at RHIC in a single-freeze-out model," Acta Physica Polonica B, vol. 35, pp. 779-798, 2004.

[13] A. Bazavov, T. Bhattacharya, M. Cheng et al., "Equation of state and QCD transition at finite temperature," Physical Review D, vol. 80, no. 1, Article ID 014504, 24 pages, 2009.

[14] S. Borsányi, Z. Fodor, C. Hoelbling et al., "Is there still any $T_{c}$ mystery in lattice QCD? Results with physical masses in the continuum limit III," Journal of High Energy Physics, vol. 2010, article 73, 2010.

[15] S. Borsanyi, Z. Fodor, S. D. Katz, S. Krieg, C. Ratti, and K. Szabo, "Fluctuations of conserved charges at finite temperature from lattice QCD," Journal of High Energy Physics, vol. 2012, p. 138, 2012.

[16] F. Becattini, P. Castorina, A. Milov, and H. Satz, "A comparative analysis of statistical hadron production," The European Physical Journal C, vol. 66, no. 3-4, pp. 377-386, 2010.

[17] P. Castorina, A. Iorio, and H. Satz, "Hadron freeze-out and Unruh radiation," http://arxiv.org/abs/1409.3104.

[18] A. Bazavov, H. T. Ding, P. Hegde et al., "Additional strange hadrons from QCD thermodynamics and strangeness freezeout in heavy ion collisions," Physical Review Letters, vol. 113, no. 7, Article ID 072001, 5 pages, 2014.

[19] J. Beringer, J. F. Arguin, R. M. Barnett et al., "Review of particle physics," Physical Review D, vol. 86, Article ID 010001, 2012.

[20] J. Stachel, A. Andronic, P. Braun-Munzinger, and K. Redlich, "Confronting LHC data with the statistical hadronization model," Journal of Physics: Conference Series, vol. 509, no. 1, Article ID 012019, 2014.

[21] J. Steinheimer, J. Aichelin, and M. Bleicher, "Nonthermal $p / \pi$ ratio at LHC as a consequence of hadronic final state interactions," Physical Review Letters, vol. 110, Article ID 042501, 2013.

[22] F. Becattini, E. Grossi, M. Bleicher, J. Steinheimer, and R. Stock, "Centrality dependence of hadronization and chemical freezeout conditions in heavy ion collisions at $\sqrt{s_{N N}}=2.76 \mathrm{TeV}$," http://arxiv.org/abs/1405.0710.

[23] M. Petráň, J. Letessier, V. Petráček, and J. Rafelski, "Hadron production and quark-gluon plasma hadronization in $\mathrm{Pb}-\mathrm{Pb}$ collisions at $\sqrt{s_{N N}}=2.76 \mathrm{TeV}$,' Physical Review C, vol. 88, Article ID 034907, 2013.

[24] S. Chatterjee, R. M. Godbole, and S. Gupta, "Strange freezeout," Physics Letters B, vol. 727, pp. 554-557, 2013.

[25] K. Bugaev, "Chemical freeze-out of strange particles and possible root of strangeness suppression," EPL, vol. 104, no. 2, Article ID 22002, 2013.

[26] S. Chatterjee and B. Mohanty, "Production of light nuclei in heavy-ion collisions within a multiple-freezeout scenario," Physical Review C, vol. 90, no. 3, Article ID 034908, 7 pages, 2014.

[27] S. Wheaton, J. Cleymans, and M. Hauer, “THERMUS-a thermal model package for ROOT," Computer Physics Communications, vol. 180, no. 1, pp. 84-106, 2009. 
[28] L. Ahle, Y. Akiba, K. Ashktorab et al., "Excitation function of $\mathrm{K}^{+}$ and $\pi^{+}$production in $\mathrm{Au}+\mathrm{Au}$ reactions at $2-10 \mathrm{AGeV}$," Physics Letters B, vol. 476, no. 1-2, pp. 1-8, 2000.

[29] J. Klay, N. N. Ajitanand, J. M. Alexander et al., "Charged pion production in $2 A$ to $8 A \mathrm{GeV}$ central $\mathrm{Au}+\mathrm{Au}$ Collisions," Physical Review C, vol. 68, no. 5, Article ID 054905, 12 pages, 2003.

[30] L. Ahle, Y. Akiba, K. Ashktorab et al., "An excitation function of $\mathrm{K}^{-}$and $\mathrm{K}^{+}$production in $\mathrm{Au}+\mathrm{Au}$ reactions at the AGS," Physics Letters B, vol. 490, no. 1-2, pp. 53-60, 2000.

[31] J. Klay, N. N. Ajitanand, J. M. Alexander et al., "Longitudinal flow of protons from (2-8)A GeV central $A u+A u$ collisions," Physical Review Letters, vol. 88, Article ID 102301, 2002.

[32] C. Blume and C. Markert, "Strange hadron production in heavy ion collisions from SPS to RHIC," Progress in Particle and Nuclear Physics, vol. 66, p. 834, 2011.

[33] B. B. Back, R. R. Betts, J. Chang et al., "Baryon Rapidity Loss in Relativistic Au+Au Collisions," Physical Review Letters, vol. 86, 1970.

[34] J. Barrette, R. Bellwied, S. Bennett et al., "Proton and pion production in $\mathrm{Au}+\mathrm{Au}$ collisions at $10.8 \mathrm{~A} \mathrm{GeV} / c$," Physical Review C, vol. 62, Article ID 024901, 2000.

[35] L. Ahle, Y. Akiba, K. Ashktorab et al., "Proton and deuteron production in $\mathrm{Au}+\mathrm{Au}$ reactions at 11.6 AGeV/c," Physical Review C, vol. 60, no. 6, Article ID 064901, 16 pages, 1999.

[36] B. B. Back, R. R. Betts, J. Chang et al., "Antilambda production in $\mathrm{Au}+\mathrm{Au}$ collisions at $11.7 \mathrm{~A} \mathrm{GeV/c}$," Physical Review Letters, vol. 87, Article ID 242301, 2001.

[37] B. B. Back, R. R. Betts, J. Chang et al., "Production of $\phi$ mesons in $\mathrm{Au}+\mathrm{Au}$ collisions at 11.7A GeV/c," Physical Review C, vol. 69, Article ID 054901, 2004.

[38] C. Alt, T. Anticic, B. Baatar et al., "Pion and kaon production in central $\mathrm{Pb}+\mathrm{Pb}$ collisions at $20 \mathrm{~A}$ and $30 \mathrm{~A} \mathrm{GeV}$ : evidence for the onset of deconfinement," Physical Review C, vol. 77, Article ID 024903, 2008.

[39] C. Alt, T. Anticic, B. Baatar et al., "Energy and centrality dependence of antiproton and proton production and the antilambda to antiproton ratio in $\mathrm{Pb}+\mathrm{Pb}$ collisions between $20 \mathrm{~A}$ $\mathrm{GeV}$ and 158A GeV," http://arxiv.org/abs/nucl-ex/0512033.

[40] C. Alt, T. Anticic, B. Baatar et al., "Energy dependence of $\lambda$ and $\xi$ production in central $\mathrm{Pb}+\mathrm{Pb}$ collisions at $20 \mathrm{~A}, 30 \mathrm{~A}, 40 \mathrm{~A}, 80 \mathrm{~A}$, and $158 \mathrm{~A} \mathrm{GeV}$ measured at the CERN Super Proton Synchrotron," Physical Review C, vol. 78, no. 3, Article ID 034918, 15 pages, 2008.

[41] C. Alt, T. Anticic, B. Baatar et al., "Energy dependence of $\phi$ meson production in central $\mathrm{Pb}+\mathrm{Pb}$ collisions at $\sqrt{s_{N N}}=6$ to 17 GeV," Physical Review C, vol. 78, Article ID 044907, 2008.

[42] S. Afanasiev, T. Anticic, D. Barna et al., "Energy dependence of pion and kaon production in central $\mathrm{Pb}+\mathrm{Pb}$ collisions," Physical Review C, vol. 66, Article ID 054902, 2002.

[43] F. Antinori, P. Bacon, A. Badalà et al., "Enhancement of hyperon production at central rapidity in $158 \mathrm{~A} \mathrm{GeV/c} \mathrm{Pb}-\mathrm{Pb}$ collisions," Journal of Physics G: Nuclear and Particle Physics, vol. 32, no. 4, pp. 427-442, 2006.

[44] I. Bearden, H. Bøggild, J. Boissevain et al., "Particle production in central $\mathrm{Pb}+\mathrm{Pb}$ collisions at $158 \mathrm{~A} \mathrm{GeV/c,"} \mathrm{Physical} \mathrm{Review} \mathrm{C,}$ vol. 66, no. 4, Article ID 044907, 9 pages, 2002.

[45] F. Antinori, P. A. Bacone, A. Badalà et al., "Energy dependence of hyperon production in nucleus-nucleus collisions at SPS," Physics Letters B, vol. 595, no. 1-4, pp. 68-74, 2004.
[46] S. Afanasev, T. Anticic, J. Bächler et al., "Production of $\varphi$ mesons in $\mathrm{p}+\mathrm{p}, \mathrm{p}+\mathrm{Pb}$ and central $\mathrm{Pb}+\mathrm{Pb}$ collisions at $\mathrm{GeV} E_{\text {beam }}$ = 158 A GeV," Physics Letters B, vol. 491, no. 1-2, pp. 59-66, 2000.

[47] L. Kumar, "STAR results from the RHIC beam energy scan-I," Nuclear Physics A, vol. 904-905, pp. 256c-263c, 2013.

[48] L. Kumar, "Identified Hadron production from the RHIC beam energy scan," Journal of Physics G, vol. 38, Article ID 124145, 2011.

[49] L. Kumar, "Centrality dependence of freeze-out parameters from $\mathrm{Au}+\mathrm{Au}$ collisions at $\sqrt{s_{N N}}=7.7,11.5$ and $39 \mathrm{GeV}$," Central European Journal of Physics, vol. 10, article 1274, 2012, http://arxiv.org/abs/1201.4203.

[50] S. Das, "Centrality dependence of freeze-out parameters from the beam energy scan at STAR," Nuclear Physics A, vol. 904-905, pp. 891c-894c, 2013.

[51] X. Zhu, "Measurements of $k_{\mathrm{S} 0}, \Lambda$ and from $\mathrm{Au}+\mathrm{Au}$ Collisions at $\sqrt{s_{N N}}=7.7,11.5$ and $39 \mathrm{GeV}$ in STAR," Acta Physica Polonica $B$, vol. 5, article 213, 2012.

[52] F. Zhao and STAR Collaboration, "Beam energy dependence of strange hadron production from STAR at RHIC," Journal of Physics: Conference Series, vol. 509, Article ID 012085, 2014.

[53] L. Kumar, "Systematics of kinetic freeze-out properties in high energy collisions from STAR," Nuclear Physics A, 2014.

[54] S. Das, "Study of freeze-out dynamics in STAR at RHIC Beam Energy Scan Program," Journal of Physics: Conference Series, vol. 509, Article ID 012066, 2014.

[55] B. I. Abelev, M. M. Aggarwal, Z. Ahammed et al., "Systematic measurements of identified particle spectra in $p p, d+A u$ and $\mathrm{Au}+\mathrm{Au}$ collisions at the STAR detector," Physical Review C, vol. 79, no. 3, Article ID 034909, 58 pages, 2009.

[56] M. Aggarwal, Z. Ahammed, A. V. Alakhverdyants et al., "Strange and multistrange particle production in $\mathrm{Au}+\mathrm{Au}$ collisions at $\sqrt{s_{N N}}=62.4 \mathrm{GeV}$," Physical Review C, vol. 83, Article ID 024901, 2011.

[57] B. Abelev, M. M. Aggarwal, Z. Ahammed et al., "Measurements of $\phi$ meson production in relativistic heavy-ion collisions at the BNL Relativistic Heavy Ion Collider (RHIC)," Physical Review C, vol. 79, Article ID 064903, 2009.

[58] K. Adcox, S. S. Adler, N. N. Ajitanand et al., "Measurement of $\Lambda$ and $\bar{\Lambda}$ particles in $\mathrm{Au}+\mathrm{Au}$ collisions at $\sqrt{s_{N N}}=130 \mathrm{GeV}$," Physical Review Letters, vol. 89, Article ID 092302, 2002.

[59] J. Adams, C. Adler, M. M. Aggarwal et al., "Multistrange Baryon production in Au-Au collisions at $\sqrt{S_{N N}}=130 \mathrm{GeV}$," Physical Review Letters, vol. 92, no. 18, Article ID 182301, 6 pages, 2004.

[60] C. Adler et al., "Midrapidity $\varphi$ production in Au+Au collisions at $\sqrt{s_{N N}}=130$ GeV," Physical Review C, vol. 65, Article ID 041901, 2002 .

[61] J. Adams, "Scaling properties of hyperon production in $\mathrm{Au}+$ Au collisions at $\sqrt{S_{N N}}=200$," Physical Review Letters, vol. 98, Article ID 062301, 2007.

[62] J. Adams, C. Adle, and M. M. Aggarwal, " $\phi$ meson production in $\mathrm{Au}+\mathrm{Au}$ and $\mathrm{p}+\mathrm{p}$ collisions at $\sqrt{S_{N N}}=200 \mathrm{GeV}$," Physics Letters $B$, vol. 612, no. 3-4, pp. 181-189, 2005.

[63] L. Milano, "Identified charged hadron production in $\mathrm{Pb}-\mathrm{Pb}$ collisions at the LHC with the ALICE Experiment," Nuclear Physics A, vol. 904-905, pp. 531c-534c, 2013.

[64] W. Reisdorf, D. Best, A. Gobbi et al., "Central collisions of Au on $\mathrm{Au}$ at 150, 250 and $400 \mathrm{~A} \cdot \mathrm{MeV}$," Nuclear Physics A, vol. 612, no. 3-4, pp. 493-556, 1997.

[65] S. Adler, C. Aidala, S. H. Aronson et al., "Systematic studies of the centrality and $\sqrt{s_{N N}}$ dependence of the $d E_{T} / d \eta$ and 
$d E_{\mathrm{ch}} / d \eta$ in heavy ion collisions at midrapidity," Physical Review $C$, vol. 71, Article ID 034908, 2005.

[66] S. Afanasiev, T. Anticic, B. Baatar et al., "Recent results on spectra and yields from NA49," Nuclear Physics A, vol. 715, pp. 161c170c, 2003.

[67] J. Bachler, J. Bächlerf, D. Barna et al., "Hadron production in nuclear collisions from the NA49 experiment at $158 \mathrm{GeV} / \mathrm{c} \cdot \mathrm{A}$," Nuclear Physics A, vol. 661, no. 1-4, pp. 45-54, 1999.

[68] R. Sahoo, "Transverse energy measurement and fluctuation studies in ultra-relativistic heavy ion collisions," http://arxiv .org/abs/0804.1800.

[69] S. Chatrchyan, V. Khachatryan, A. M. Sirunyan et al., "Measurement of the pseudorapidity and centrality dependence of the transverse energy density in $\mathrm{Pb}-\mathrm{Pb}$ collisions at $\sqrt{S_{N N}}=$ 2.76 TeV," Physical Review Letters, vol. 109, no. 15, Article ID 152303, 16 pages, 2012.

[70] C. Muentz, "Recent results from E866 at BNL," http://arxiv.org/ abs/nucl-ex/9806002.

[71] C. Alt, "Energy and centrality dependence of $\bar{p}$ and $p$ production and the $\bar{\Lambda} / \bar{p}$ ratio in $\mathrm{Pb}+\mathrm{Pb}$ collisions between $20 \mathrm{~A} \mathrm{GeV}$ and 158A GeV," Physical Review C, vol. 73, Article ID 044910, 2006.

[72] B. Abelev, J. Adam, and D. Adamová, "Centrality dependence of $\pi, K$, and $P$ production in $\mathrm{Pb}-\mathrm{Pb}$ collisions at $\sqrt{s_{N N}}=2.76$ TeV," Physical Review C, vol. 88, Article ID 044910, 2013.

[73] L. Kumar and STAR Collaboration, "Bulk properties in $\mathrm{Au}+\mathrm{Au}$ collisions $\sqrt{s_{N N}}=9.2 \mathrm{GeV}$ in STAR experiment at RHIC," Nuclear Physics A, vol. 830, no. 1-4, pp. 275c-278c, 2009.

[74] B. Mohanty, "QCD phase diagram: phase transition, critical point and fluctuations," Nuclear Physics A, vol. 830, no. 1-4, pp. 899c-907c, 2009.

[75] B. I. Abelev, M. Planinić, N. Poljak et al., "Identified particle production, azimuthal anisotropy, and interferometry measurements in $\mathrm{Au}+\mathrm{Au}$ collisions at $\sqrt{s_{N N}}=9.2 \mathrm{GeV}$," Physical Review C, vol. 81, Article ID 024911, 2010.

[76] L. Kumar, "Results from the STAR beam energy scan program," Nuclear Physics A, vol. 862-863, pp. 125-131, 2011.

[77] M. Aggarwal, Z. Ahammed, A. V. Alakhverdyants et al., "An experimental exploration of the QCD phase diagram: the search for the critical point and the onset of de-confinement," http://arxiv.org/abs/1007.2613.

[78] L. Kumar, "Review of recent results from the RHIC beam energy scan," Modern Physics Letters A, vol. 28, no. 36, Article ID 1330033, 17 pages, 2013.

[79] J. Cleymans, H. Oeschler, K. Redlich, and S. Wheaton, "Transition from baryonic to mesonic freeze-out," Physics Letters B, vol. 615, no. 1-2, pp. 50-64, 2005.

[80] F. Becattini, "Remark on statistical model fits to particle ratios in relativistic heavy ion collisions," http://arxiv.org/abs/0707.4154.

[81] J. Cleymans, H. Oeschler, K. Redlich, and S. Wheaton, "Comparison of chemical freeze-out criteria in heavy-ion collisions," Physical Review C, vol. 73, Article ID 034905, 2006.

[82] A. Andronic, P. Braun-Munzinger, and J. Stachel, "The Horn, the hadron mass spectrum and the QCD phase diagram: the statistical model of hadron production in central nucleus-nucleus collisions," Nuclear Physics A, vol. 834, pp. 237C-240C, 2010.

[83] V. Begun, W. Florkowski, and M. Rybczynski, "Transversemomentum spectra of strange particles produced in $\mathrm{Pb}+\mathrm{Pb}$ collisions at $\sqrt{s_{N N}}=2.76 \mathrm{TeV}$ in the chemical non-equilibrium model," http://arxiv.org/abs/1405.7252.

[84] S. Chatterjee, R. Godbole, and S. Gupta, in preparation.
[85] A. Andronic, P. Braun-Munzinger, J. Stachel, and H. Stocker, "Production of light nuclei, hypernuclei and their antiparticles in relativistic nuclear collisions," Physics Letters B, vol. 697, no. 3, pp. 203-207, 2011.

[86] J. Cleymans, S. Kabana, I. Kraus, H. Oeschler, K. Redlich, and N. Sharma, "Antimatter production in proton-proton and heavyion collisions at ultrarelativistic energies," Physical Review C, vol. 84, no. 5, Article ID 054916, 2011.

[87] M. Jacob and P. Landshoff, "Minijets: origin and usefulness," Modern Physics Letters A, vol. 1, p. 657, 1986.

[88] X.-N. Wang, "A pQCD-based approach to parton production and equilibration in high-energy nuclear collisions," Physics Reports, vol. 280, no. 5-6, pp. 287-371, 1997.

[89] T. Abbott, L. Ahle, Y. Akiba et al., "Systematics of midrapidity transverse energy distributions in limited apertures from $P+B e$ to $\mathrm{Au}+\mathrm{Au}$ collisions at relativistic energies," Physical Review $\mathrm{C}$, vol. 63, Article ID 064602, 2001.

[90] J. Adams, J. K. Adkins, G. Agakishiev et al., "Measurements of transverse energy distributions in $\mathrm{Au}+\mathrm{Au}$ collisions at $\sqrt{s_{N N}}=$ 200 GeV," Physical Review C, vol. 70, Article ID 054907, 2004.

[91] J. Cleymans, R. Sahoo, D. Mahapatra, D. Srivastava, and S. Wheaton, "Transverse energy per charged particle and freezeout criteria in heavy-ion collisions," Physics Letters B, vol. 660, no. 3, pp. 172-175, 2008.

[92] J. Cleymans, R. Sahoo, D. Mahapatra, D. Srivastava, and S. Wheaton, "Saturation of $E_{T} / N_{c h}$ and freeze-out criteria in heavy-ion collisions," Journal of Physics G, vol. 35, no. 10, Article ID 104147, 2008.

[93] D. Prorok, “The transverse energy per charged particle estimates in the framework of a statistical model," Nuclear Physics A, vol. 749, pp. 194-197, 2005.

[94] M. Gyulassy, "Lectures on the theory of high energy A+A at RHIC," Lecture Notes in Physics, vol. 583, pp. 37-79, 2002.

[95] P. Petreczky, "Viewpoint: new measurements of the most perfect liquid," Physics, vol. 5, article 112, 2012.

[96] R. Sahoo and A. N. Mishra, "Transverse energy and charged particle production in heavy-ion collisions: from RHIC to LHC," International Journal of Modern Physics E, vol. 23, no. 4, Article ID 1450024, 19 pages, 2014.

[97] J. Cleymans and K. Redlich, "Unified description of freeze-out parameters in relativistic heavy ion collisions," Physical Review Letters, vol. 81, article 5284, 1998.

[98] P. Braun-Munzinger and J. Stachel, "Particle ratios, equilibration, and the QCD phase boundary," Journal of Physics G, vol. 28, no. 7, p. 1971, 2002.

[99] A. Tawfik, "Influence of strange quarks on the QCD phase diagram and chemical freeze-out," Journal of Physics G: Nuclear and Particle Physics, vol. 31, no. 6, Article ID S1105, 2005.

[100] L. van Hove, "Multiplicity dependence of $p_{t}$ spectrum as a possible signal for a phase transition in hadronic collisions," Physics Letters B, vol. 118, no. 1-3, pp. 138-140, 1982.

[101] B. Abelev, J. Adam, D. Adamová et al., "Multiplicity dependence of the average transverse momentum in $\mathrm{pp}, \mathrm{p}-\mathrm{Pb}$, and $\mathrm{Pb}-\mathrm{Pb}$ collisions at the LHC," Physics Letters B, vol. 727, no. 4-5, pp. 371-380, 2013.

[102] B. Abelev, J. Adam, D. Adamová et al., "Pion, kaon, and proton production in central $\mathrm{Pb}-\mathrm{Pb}$ collisions at $\sqrt{s_{N N}}=2.76 \mathrm{TeV}$," Physical Review Letters, vol. 109, Article ID 252301, 15 pages, 2012. 

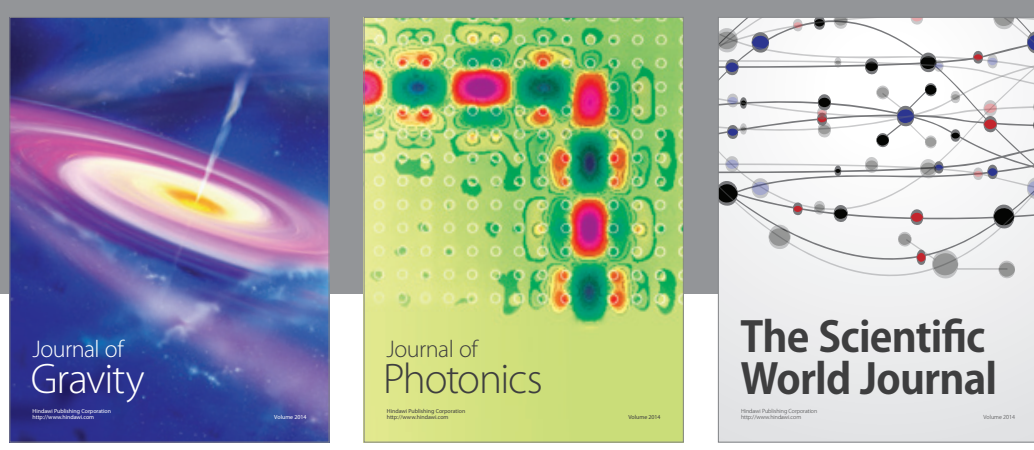

The Scientific World Journal
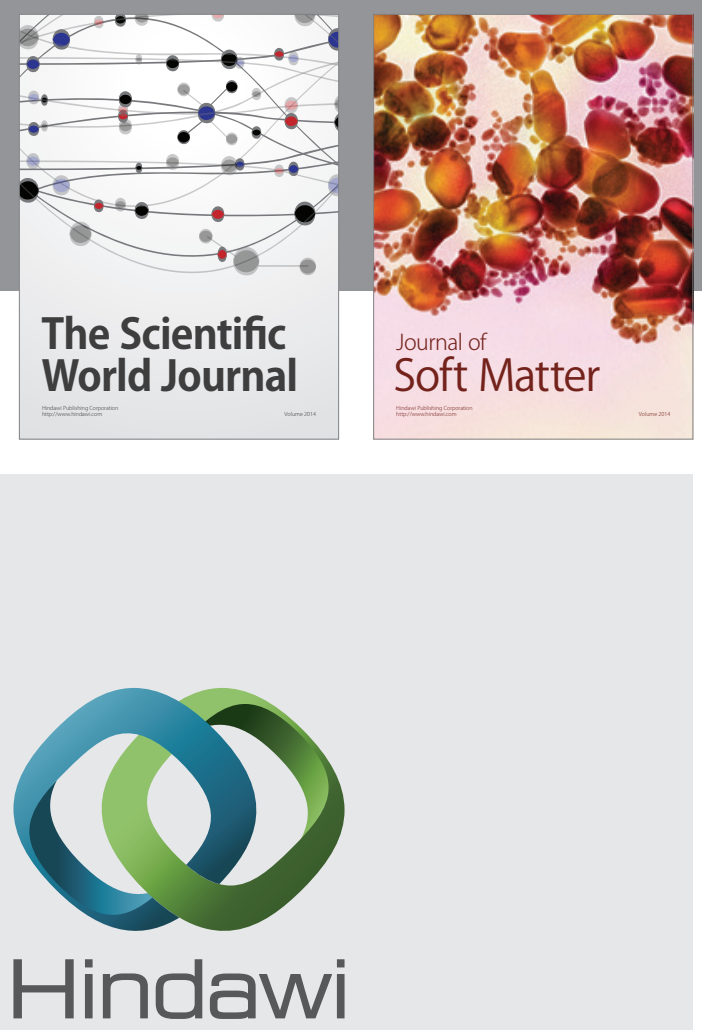

Submit your manuscripts at

http://www.hindawi.com

nternational Journal of

Statistical Mechanics
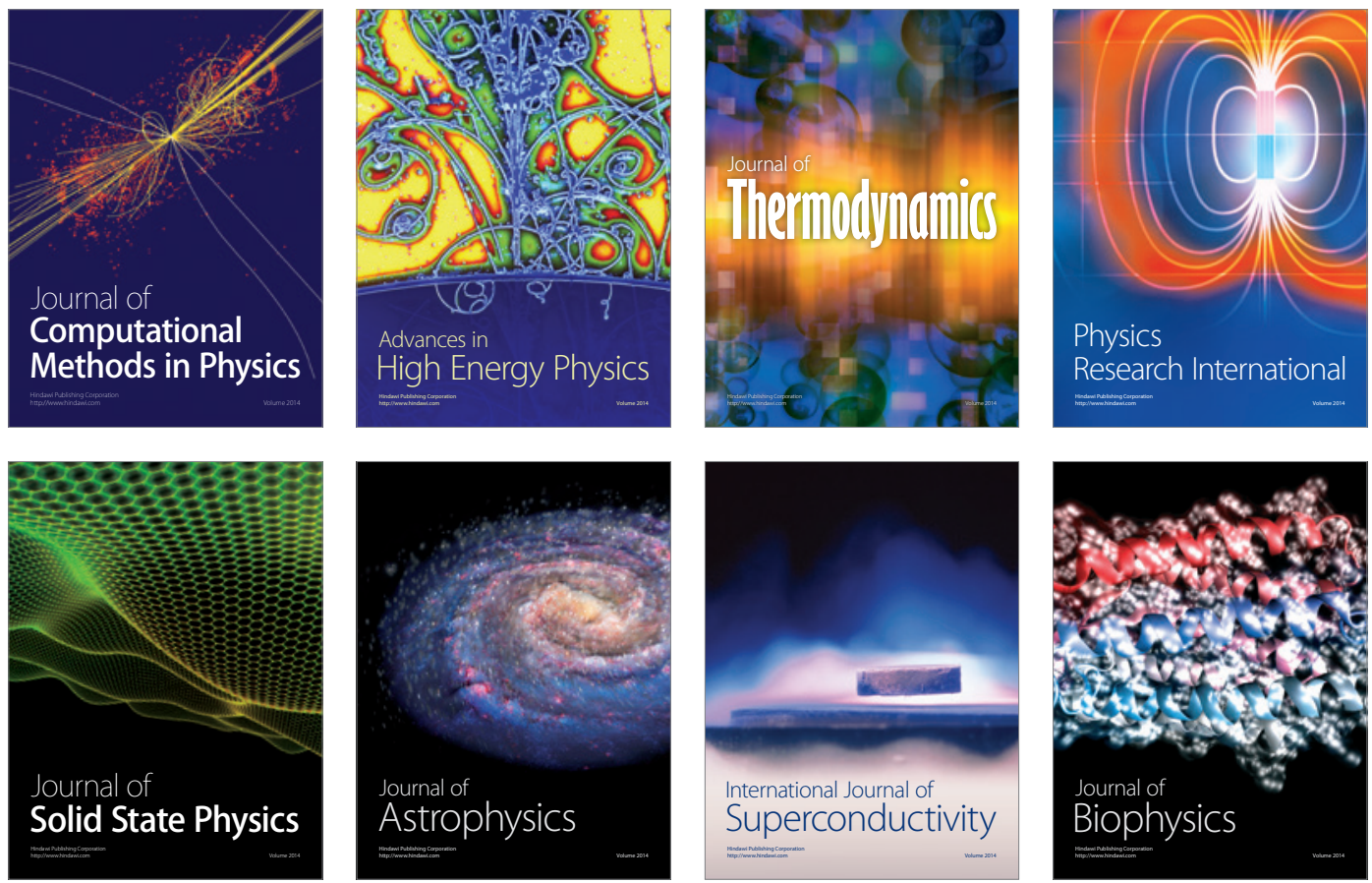
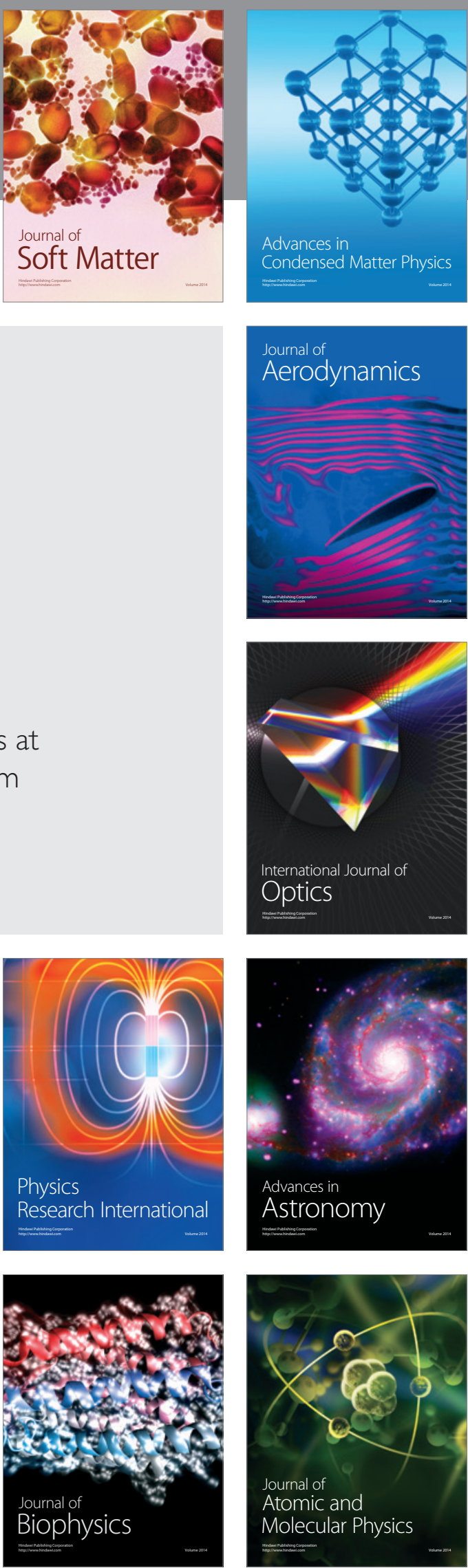